\title{
REGULARITY OF SOLUTIONS IN PLASTICITY. I: CONTINUUM
}

Abstract. The aim of this paper is to study the problem of regularity of solutions in Hencky plasticity. We consider a non-homogeneous material whose elastic-plastic properties change discontinuously. We prove that the displacement solutions belong to the space $L D(\Omega) \equiv\left\{\mathbf{u} \in L^{1}\left(\Omega, \mathbb{R}^{n}\right) \mid\right.$ $\left.\nabla \mathbf{u}+(\nabla \mathbf{u})^{T} \in L^{1}\left(\Omega, \mathbb{R}^{n \times n}\right)\right\}$ if the stress solution is continuous and belongs to the interior of the set of admissible stresses, at each point. The part of the functional which describes the work of boundary forces is relaxed.

1. Introduction. The principal aim of this contribution is to prove a theorem on regularity of displacement solutions in Hencky plasticity (see Theorem 21). We consider a non-homogeneous material whose elastic-plastic properties change discontinuously. We prove that the displacement solutions belong to the space $L D(\Omega)$ if the stress solution is continuous and belongs to the interior of the set of admissible stresses, at each point. The part of the functional which describes the work of boundary forces is relaxed.

In [1] (resp. [5]) the existence of solutions for the relevant integral functional is proved in the space $S B V(\Omega)$ of special vector fields with bounded variation (resp. $S B D(\Omega)$ of special vector fields with bounded deformation). Those authors assume that the potential has nonlinear growth at infinity.

In [17] the problem of regularity of displacement solutions, in a homogeneous Hencky material with the von Mises yield criterion, is investigated. The proof of the main theorem of [17] (Theorem 5.1) is based on the relation between the displacement field and the associated stress tensor (cf. formula (1.8) of [17]). But the formula (1.8) describes the relation between the dis-

2000 Mathematics Subject Classification: Primary 49N60; Secondary 49J45, 49K30, $74 \mathrm{C} 05$. tion.

Key words and phrases: Hencky plasticity, displacement solution, bounded deforma-

Supported by Committee for Scientific Research (Poland) grant No. 5P03A04620. 
placement solution and the stress solution only in the case when the space of admissible stress fields is given by the inequality $\left(\sum_{i, j=1}^{n}\left|\sigma_{i j}^{D}\right|^{2}\right)^{1 / 2} \leq k$ (see the Prandtl-Reuss law of plasticity [19, formula (2.10b)]). Moreover, the authors do not consider bodies clamped on the boundary (or on part of the boundary).

Seregin [22] investigates the local continuity of the stress and displacement solution in a homogeneous Hencky material under the assumption of regularity of the volume forces. He considers the problem only for displacements which satisfy the boundary condition exactly. Therefore, there is no study of the relaxation of the displacement boundary condition.

Anzellotti and Giaquinta [3] study the local regularity of the minimizers of the functionals defined on the space $B V(\Omega)$. They obtain the regularity property of the minimizers under the assumption that the normal integrand

$$
\Omega \times \mathbb{R}^{n \times n} \ni(x, \mathbf{p}) \mapsto j(x, \mathbf{p}) \in \mathbb{R} \cup\{+\infty\}
$$

is of class $C^{2}$ with respect to $\mathbf{p}$, and is continuous with respect to the first variable. They do not consider boundary conditions.

In [10] the problem of regularity of solutions for a static plate is studied.

Kohn and Temam [18] solve the existence problem for an elastic-perfectly plastic solid made of a homogeneous and isotropic Hencky material. To prove that the functional of the total potential energy is weak* lower semicontinuous (l.s.c.) in the space $B D(\Omega)$, they use the method of relaxation of the kinematic boundary condition (see also [23]).

The existence problem for an anisotropic elastic-perfectly plastic solid made of a non-homogeneous Hencky material, with the Signorini constraints on the boundary, is solved in [6]. The Signorini problem for an isotropic homogeneous body made of a Hencky material is solved in [26].

2. Some basic definitions and theorems. Let $\Omega$ be a bounded, open, connected set of class $C^{1}$ in $\mathbb{R}^{n}$. The space of continuous functions with compact support is denoted by $C_{c}$. Let $C^{\infty}\left(\Omega, \mathbb{R}^{m}\right)$ be the space of $\mathbb{R}^{m}$ valued, infinitely differentiable functions. Moreover, the space of infinitely differentiable functions equal to 0 at the boundary $\operatorname{Fr} \Omega$ of $\Omega$ is denoted by $C_{0}^{\infty}(\Omega)$. Finally, $\mathbb{M}_{b}\left(\Omega, \mathbb{R}^{m}\right)$ is the space of $\mathbb{R}^{m}$-valued, Radon, bounded, regular measures on $\Omega$, with the norm $\|\cdot\|_{\mathbb{M}_{b}\left(\Omega, \mathbb{R}^{m}\right)}$.

We will use the duality pairs $\left(\mathbb{M}_{r}, C_{c}\right)$ or $\left(\mathbb{M}_{b}, C_{0}\right)$, where $\mathbb{M}_{r}$ is the space of regular measures. Duality pairings will be denoted by $\langle\cdot, \cdot\rangle$, and the scalar product of $\mathbf{z}, \mathbf{z}^{*} \in \mathbb{R}^{n}$ by $\mathbf{z} \cdot \mathbf{z}^{*}$ or $\mathbf{z z}^{*}$. The scalar product of $\mathbf{w}, \mathbf{w}^{*} \in \mathbb{R}^{n \times n}$ is denoted by $\mathbf{w}: \mathbf{w}^{*}=w^{i j} w_{i j}^{*}$. Let $\mathbf{g}=\left(g_{1}, \ldots, g_{m}\right) \in C\left(\bar{\Omega}, \mathbb{R}^{m}\right)$ and $\boldsymbol{\mu}=\left(\mu_{1}, \ldots, \mu_{m}\right) \in \mathbb{M}_{b}\left(\Omega, \mathbb{R}^{m}\right)$. Then $\int_{\Omega} \mathbf{g} \cdot \boldsymbol{\mu}=\int_{\Omega} \mathbf{g} \boldsymbol{\mu} \equiv \sum_{i=1}^{m} \int_{\Omega} g_{i} \mu_{i}$. If $F:$ $Y \rightarrow \mathbb{R} \cup\{+\infty\}$, then $F^{*}$ denotes its polar function $F^{*}\left(y^{*}\right) \equiv \sup \left\{\left\langle y^{*}, y\right\rangle-\right.$ $F(y) \mid y \in Y\}$ and $\operatorname{dom} F \equiv\{y \in Y \mid F(y)<\infty\}$ is the effective domain 
of $F$ (see [12]). If $Q$ is a subset of $Y$, then $I_{Q}(\cdot)$ stands for its indicator function (taking the value 0 in $Q$ and $+\infty$ elsewhere), and $I_{Q}^{*}(\cdot)$ stands for its support function.

Finally, we need the following notations. Let $V$ be a metric space. Then $B_{V}(\Xi, r)$ is the closed ball in $V$ with center $\Xi$ and radius $r$. Furthermore, $\operatorname{cl}_{V}(Z)$ stands for the closure of $Z \subset V$ in the topology of the space $V$; analogously, $\mathrm{cl}_{\|\cdot\|}(Z)$ is the closure of the set $Z$ in the norm $\|\cdot\|$. Similarly int $Z$ denotes the interior of $Z$. We will also consider the spaces $\mathbf{E}^{n}$ of real $n \times n$ matrices and $\mathbf{E}_{s}^{n}$ of symmetric real $n \times n$ matrices. We set $\left\|\left[e_{i j}\right]\right\|_{\mathbf{E}^{n}} \equiv$ $\sum_{i, j=1}^{n}\left|e_{i j}\right|$ and $\|\cdot\|_{\mathbf{E}_{s}^{n}} \equiv\|\cdot\|_{\mathbf{E}^{n}}$. We denote by $\otimes\left(\right.$ resp. $\left.\otimes_{s}\right)$ the tensor product (resp. symmetric tensor product). Let $\mathcal{L}^{0}\left(\Omega, \mathbb{R}^{m}\right)_{\mu}$ be the set of $\mu$-measurable functions from $\Omega$ into $\mathbb{R}^{m}$. If $\tau \subset 2^{X}$ is a linear topology in a vector space $X$, then $[X, \tau]$ denotes the topological space and $[X, \tau]^{*}$ is the space dual to $[X, \tau]$. We define the following Banach spaces (see [18], [23], $[24])$ :

$$
\begin{aligned}
L D(\Omega) \equiv\left\{\mathbf{u} \in L^{1}\left(\Omega, \mathbb{R}^{n}\right) \mid\right. \\
\left.\varepsilon_{i j}(\mathbf{u})=\frac{1}{2}\left(\frac{\partial u_{i}}{\partial x_{j}}+\frac{\partial u_{j}}{\partial x_{i}}\right) \in L^{1}(\Omega), i, j=1, \ldots, n\right\}, \\
B D(\Omega) \equiv\left\{\mathbf{u} \in L^{1}\left(\Omega, \mathbb{R}^{n}\right) \mid \varepsilon_{i j}(\mathbf{u}) \in \mathbb{M}_{b}(\Omega), i, j=1, \ldots, n\right\},
\end{aligned}
$$

with the natural norms

$$
\|\mathbf{u}\|_{L D}=\|\mathbf{u}\|_{L^{1}}+\sum_{i, j}^{n}\left\|\varepsilon_{i j}(\mathbf{u})\right\|_{L^{1}}, \quad\|\mathbf{u}\|_{B D}=\|\mathbf{u}\|_{L^{1}}+\sum_{i, j}^{n}\left\|\varepsilon_{i j}(\mathbf{u})\right\|_{\mathbb{M}_{b}} .
$$

Moreover, $\mathcal{R}_{0} \equiv\{\mathbf{u} \in B D(\Omega) \mid \boldsymbol{\varepsilon}(\mathbf{u})=\mathbf{0}\}$ denotes the space of rigid motions in $\mathbb{R}^{n}$.

Proposition 1 (see [23]). Let $B D(\Omega)$ and $L^{1}\left(\operatorname{Fr} \Omega, \mathbb{R}^{n}\right)$ be endowed with the norm topologies. There exists a continuous surjective linear trace $\gamma_{B}$ from $B D(\Omega)$ into $L^{1}\left(\operatorname{Fr} \Omega, \mathbb{R}^{n}\right)$ such that $\gamma_{B}(\mathbf{u})=\mathbf{u}_{\mid \operatorname{Fr} \Omega}$ for all $\mathbf{u} \in$ $B D \cap C\left(\bar{\Omega}, \mathbb{R}^{n}\right)$.

We define spaces

$$
X \equiv C_{c}\left(\Omega, \mathbb{R}^{n}\right) \times C_{c}\left(\Omega, \mathbf{E}_{s}^{n}\right), \quad X_{0} \equiv\{(\mathbf{g}, \mathbf{h}) \in X \mid \mathbf{g}=\operatorname{div} \mathbf{h}\},
$$

endowed with the natural norm

$$
\begin{aligned}
\|\mathbf{g}\|_{C\left(\Omega, \mathbb{R}^{n}\right)} & +\|\mathbf{h}\|_{C\left(\Omega, \mathbf{E}_{s}^{n}\right)} \\
& \equiv \sup \left\{\|\mathbf{g}(x)\|_{\mathbb{R}^{n}} \mid x \in \Omega\right\}+\sup \left\{\|\mathbf{h}(x)\|_{\mathbf{E}_{s}^{n}} \mid x \in \Omega\right\}
\end{aligned}
$$

for $\mathbf{g} \in C\left(\Omega, \mathbb{R}^{n}\right)$ and $\mathbf{h} \in C\left(\Omega, \mathbf{E}_{s}^{n}\right)$. Then $B D(\Omega)$ is isomorphic to the dual of $\left[X / X_{0},\|\cdot\|_{C\left(\Omega, \mathbb{R}^{n}\right)}+\|\cdot\|_{C\left(\Omega, \mathbf{E}_{s}^{n}\right)}\right]$ (see [23] and [24]). 
The topology $\sigma\left(\left(X / X_{0}\right)^{*}, X\right)=\sigma\left(B D(\Omega), C_{c}\left(\Omega, \mathbb{R}^{n}\right) \times C_{c}\left(\Omega, \mathbf{E}_{s}^{n}\right)\right)$ is called the weak ${ }^{*} B D$ topology. A net $\left\{\mathbf{u}_{\delta}\right\}_{\delta \in D} \subset B D(\Omega)$ is convergent to $\mathbf{u}_{0} \in B D(\Omega)$ in this topology if and only if for all $(\mathbf{g}, \mathbf{h}) \in X$,

$$
\int_{\Omega} \mathbf{g} \cdot\left(\mathbf{u}_{0}-\mathbf{u}_{\delta}\right) d x+\int_{\Omega} \mathbf{h}: \varepsilon\left(\mathbf{u}_{0}-\mathbf{u}_{\delta}\right) \rightarrow 0
$$

(see [13, pp. 73-81] and [11, pp. 26-29]). For every $\varphi \in L^{1}\left(\operatorname{Fr} \Omega, \mathbb{R}^{n}\right)$, the set $\left\{\mathbf{u} \in B D(\Omega) \mid \boldsymbol{\gamma}_{B}(\mathbf{u})=\boldsymbol{\varphi}\right\}$ is dense in the space $\left[B D(\Omega)\right.$, weak ${ }^{*}$ topology] (see [6, Proposition 2.5]). Then the trace operator $\gamma_{B}$ is not continuous on $\left[B D(\Omega)\right.$, weak ${ }^{*}$ topology] if the space $L^{1}\left(\operatorname{Fr} \Omega, \mathbb{R}^{n}\right)$ is endowed with a Hausdorff topology (or a $T_{1}$-topology, see [13, Chap. 1, Sec. 5] and [23]).

Definition 1 (see [23] and [13, Chap. 1, Sec. 6]). A net $\left\{\mathbf{u}_{\delta}\right\}_{\delta \in D}$ converges to $\mathbf{u}_{0}$ (in the topology $(2.7)-(2.8)$ ) if

$$
\begin{aligned}
& \mathbf{u}_{\delta} \rightarrow \mathbf{u}_{0} \quad \text { in }\|\cdot\|_{L^{p}\left(\Omega, \mathbb{R}^{n}\right)} \forall p \text { such that } 1 \leq p<q=n /(n-1) \\
& \text { and weakly in } L^{q}\left(\Omega, \mathbb{R}^{n}\right) \text { (if } n=1 \text { then } q=\infty \text { ), } \\
& \text { (2.8) } \quad \varepsilon\left(\mathbf{u}_{\delta}\right) \rightarrow \varepsilon\left(\mathbf{u}_{0}\right) \text { weak* in } \mathbb{M}_{b}\left(\Omega, \mathbf{E}_{s}^{n}\right) \text {. }
\end{aligned}
$$

Proposition 2 (cf. [6]). The weak $k^{*} B D(\Omega)$ topology and the topology (2.7)-(2.8) are equivalent on bounded subsets of $B D(\Omega)$.

Proof. Every bounded net $\left\{\mathbf{u}_{\delta}\right\}_{\delta \in D}$ in $B D$ contains a finer net, convergent in (2.7)-(2.8) (see [23]). Then $\mathrm{cl}_{\|\cdot\|_{B D}} B(0, r)$ is a compact set in (2.7)(2.8) and in the weak* $B D$ topology. Moreover, the weak* $B D$ topology is weaker than the (2.7)-(2.8) topology, and among all Hausdorff topologies, compact topologies are minimal (see [13, Corollary 3.1.14]).

The injection of $\left[B D(\Omega)\right.$, weak $\left.{ }^{*}\right]$ into $\left[L^{p}\left(\Omega, \mathbb{R}^{n}\right)\right.$, weak topology $]$ is continuous on bounded subsets of $B D(\Omega)$, where $1 \leq p \leq q=n /(n-1)(q=\infty$ if $n=1)$.

We define the Banach space of measurable functions

$$
W^{n}(\Omega, \operatorname{div}) \equiv\left\{\boldsymbol{\sigma} \in L^{\infty}\left(\Omega, \mathbf{E}_{s}^{n}\right) \mid \operatorname{div} \boldsymbol{\sigma} \in L^{n}\left(\Omega, \mathbb{R}^{n}\right)\right\}
$$

endowed with the natural norm

$$
\|\boldsymbol{\sigma}\|_{W^{n}(\Omega, \operatorname{div})}=\|\boldsymbol{\sigma}\|_{L^{\infty}\left(\Omega, \mathbf{E}_{s}^{n}\right)}+\|\operatorname{div} \boldsymbol{\sigma}\|_{L^{n}\left(\Omega, \mathbb{R}^{n}\right)}
$$

(cf. [23, Chapter 2, Section 7] and [6]). The distribution $\boldsymbol{\sigma}: \boldsymbol{\varepsilon}(\mathbf{u})$, where $\boldsymbol{\sigma} \in W^{n}(\Omega, \operatorname{div}), \mathbf{u} \in B D(\Omega)$, defined (for every $\varphi_{1} \in C_{c}^{\infty}(\Omega)$ ) by

$$
\left\langle\boldsymbol{\sigma}: \boldsymbol{\varepsilon}(\mathbf{u}), \varphi_{1}\right\rangle_{D^{\prime} \times D}=-\int_{\Omega}(\operatorname{div} \boldsymbol{\sigma}) \cdot \mathbf{u} \varphi_{1} d x-\int_{\Omega} \boldsymbol{\sigma}:\left(\mathbf{u} \otimes \operatorname{grad} \varphi_{1}\right) d x
$$

is a bounded measure on $\Omega$, and it is absolutely continuous with respect to $|\varepsilon(\mathbf{u})|($ see $[23])$.

Assumption 1. Let $\Omega$ and $\Omega_{1}$ be bounded open connected sets of class $C^{1}$ in $\mathbb{R}^{n}$. Moreover, let $\Omega \subset \subset \Omega_{1}$. 
TheOREm 3 (cf. [23]). There exists a continuous, linear, surjective, open map $\boldsymbol{\beta}_{B}$ from $\left[W^{n}(\Omega, \operatorname{div}),\|\cdot\|_{W^{n}(\Omega, \text { div })}\right]$ onto $\left[L^{\infty}\left(\operatorname{Fr} \Omega, \mathbb{R}^{n}\right),\|\cdot\|_{L^{\infty}}\right]$ such that for every $\boldsymbol{\sigma} \in C\left(\bar{\Omega}, \mathbf{E}_{s}^{n}\right), \boldsymbol{\beta}_{B}(\boldsymbol{\sigma})=\boldsymbol{\sigma}_{\mid \operatorname{Fr} \Omega} \cdot \boldsymbol{\nu}$, where $\boldsymbol{\nu}$ denotes the exterior unit vector normal to $\operatorname{Fr} \Omega$. Furthermore, for all $\mathbf{u} \in B D(\Omega)$ and all $\boldsymbol{\sigma} \in W^{n}(\Omega$, div), the following Green formula holds:

$$
\int_{\Omega} \boldsymbol{\sigma}: \varepsilon(\mathbf{u})+\int_{\Omega}(\operatorname{div} \boldsymbol{\sigma}) \cdot \mathbf{u} d x=\int_{\operatorname{Fr} \Omega} \boldsymbol{\beta}_{B}(\boldsymbol{\sigma}) \cdot \boldsymbol{\gamma}_{B}(\mathbf{u}) d s .
$$

REMARK 1 (see [6, Lemma 2.13]). For all $\boldsymbol{\sigma} \in W^{n}(\Omega$, div) there exists $\boldsymbol{\sigma}_{1} \in W^{n}\left(\Omega_{1}, \operatorname{div}\right)$ such that $\boldsymbol{\sigma}_{1 \mid \Omega}=\boldsymbol{\sigma}$.

3. Auxiliary theorems and spaces. In this paper, the Lebesgue and Hausdorff measures on $\Omega$ and $\operatorname{Fr} \Omega$ are denoted by $d x$ and $d s$, respectively. Let $\Gamma_{0}$ and $\Gamma_{1}\left(\Gamma_{1}=\bar{\Gamma}_{1}\right)$ be Borel subsets of $\operatorname{Fr} \Omega$ such that $\Gamma_{0} \cap \Gamma_{1}=\emptyset$ and $d s\left(\operatorname{Fr} \Omega-\left(\Gamma_{0} \cup \Gamma_{1}\right)\right)=0$. We will consider an elastic-perfectly plastic body, occupying the given set $\Omega$. We first introduce some functions. Let $\mathcal{K}: \bar{\Omega} \rightarrow 2^{\mathbf{E}_{s}^{n}}$ be a multifunction.

Assumption 2 (cf. [6], [8, p. 401] and [15, p. 19]). For every $y \in \bar{\Omega}$,

$$
\begin{aligned}
\mathcal{K}(y)=\left\{\mathbf{z}(y) \mid \mathbf{z} \in C\left(\bar{\Omega}, \mathbf{E}_{s}^{n}\right), \mathbf{z}_{\mid \mathrm{int} \Omega} \in W^{n}(\Omega, \operatorname{div}),\right. \\
\quad \mathbf{z}(x) \in \mathcal{K}(x) \text { for } d x \text {-a.e. } x \in \Omega\} .
\end{aligned}
$$

Moreover, for all $x \in \bar{\Omega}, \mathcal{K}(x)$ is a convex and closed subset in $\mathbf{E}_{s}^{n}$.

The set $\mathcal{K}(x)$ is the elasticity convex domain at the point $x$.

Let $\emptyset \neq \mathcal{K}_{1} \subset \mathcal{K}_{2}$ be convex closed subsets in $\mathbf{E}_{s}^{n}$. Moreover, let $\bar{\Omega}=\widetilde{\Omega}_{1} \cup$ $\widetilde{\Omega}_{2}, \widetilde{\Omega}_{1} \cap \widetilde{\Omega}_{2}=\emptyset, \widetilde{\Omega}_{2}=\operatorname{int} \widetilde{\Omega}_{2}$ (interior with respect to $\bar{\Omega}$ ) and $\widetilde{\Omega}_{1}=\mathrm{clint} \widetilde{\Omega}_{1}$. Then the multifunction $\mathcal{K}_{s}$, defined by $\mathcal{K}_{s}(x)=\mathcal{K}_{1}$ if $x \in \widetilde{\Omega}_{1}$ and $\mathcal{K}_{s}(x)=\mathcal{K}_{2}$ if $x \in \widetilde{\Omega}_{2}$, satisfies Assumption 2. From (3.1) we see that if $\mathbf{z}(x) \in \mathcal{K}(x)$ a.e. in $\Omega$ and $\mathbf{z} \in C\left(\bar{\Omega}, \mathbf{E}_{s}^{n}\right), \mathbf{z}_{\mid \text {int } \Omega} \in W^{n}(\Omega, \operatorname{div})$ then $\mathbf{z}(x) \in \mathcal{K}(x)$ for every $x \in \bar{\Omega}$.

Assumption 3. There exists $r_{1}>0$ such that $B_{\mathbf{E}_{s}^{n}}\left(0, r_{1}\right) \subset \mathcal{K}(x)$ for every $x \in \bar{\Omega}$. Moreover, there exist $\bar{a}>0,\left[q_{i j}\right] \in L^{\infty}\left(\Omega, \mathbf{E}_{s}^{n}\right)$ and $a_{i j k l} \in$ $L^{\infty}(\Omega, \mathbb{R})$ for $i, j, k, l \in\{1, \ldots, n\}$ such that

$$
\begin{gathered}
\sum_{i, j, k, l=1}^{n} a_{i j k l}(x) w_{i j}^{*} w_{k l}^{*}>\bar{a}\left\|\left[w_{i j}^{*}\right]\right\|_{\mathbf{E}_{s}^{n}}^{2}, \\
j^{*}\left(x,\left[w_{i j}^{*}\right]\right)=\sum_{i, j, k, l=1}^{n} a_{i j k l}(x)\left(w_{i j}^{*}-q_{i j}(x)\right)\left(w_{k l}^{*}-q_{k l}(x)\right)+I_{\mathcal{K}(x)}\left(\left[w_{i j}^{*}\right]\right)
\end{gathered}
$$

for $d x$-a.e. $x \in \Omega$ and for every $\left[w_{i j}^{*}\right] \in \mathbf{E}_{s}^{n}$. 
We define

$$
j(x, \mathbf{w}) \equiv j^{* *}(x, \mathbf{w}) \equiv \sup \left\{\mathbf{w}: \mathbf{w}^{*}-j^{*}\left(x, \mathbf{w}^{*}\right) \mid \mathbf{w}^{*} \in \mathbf{E}_{s}^{n}\right\}
$$

for $d x$-a.e. $x \in \Omega$ and all $\mathbf{w} \in \mathbf{E}_{s}^{n}$. By Assumption 3 there exists $k>0$ such that

$$
c_{n} r_{1}\|\mathbf{w}\|_{\mathbf{E}_{s}^{n}}-k \leq j(x, \mathbf{w}) \quad \text { for } d x \text {-a.e. } x \in \Omega,
$$

where the positive constant $c_{n}$ depends only on $n$ (cf. definition of the norm $\|\cdot\|_{\mathbf{E}_{s}^{n}}$ in Section 2). Define $j_{\infty}: \bar{\Omega} \times \mathbf{E}_{s}^{n} \rightarrow \mathbb{R} \cup\{+\infty\}$ by

$$
j_{\infty}(x, \mathbf{w}) \equiv \sup \left\{\mathbf{w}: \mathbf{w}^{*}-I_{\mathcal{K}(x)}\left(\mathbf{w}^{*}\right) \mid \mathbf{w}^{*} \in \mathbf{E}_{s}^{n}\right\}
$$

for $x \in \bar{\Omega}$ and $\mathbf{w} \in \mathbf{E}_{s}^{n}$. Because of Assumption 3 we have

$$
c_{n} r_{1}\|\mathbf{w}\|_{\mathbf{E}_{s}^{n}} \leq j_{\infty}(x, \mathbf{w}), \quad \forall x \in \bar{\Omega},
$$

where the positive constant $c_{n}$ depends only on $n$.

Let $\mathbf{f} \in L^{n}\left(\Omega, \mathbb{R}^{n}\right)$ and $\mathbf{g} \in L^{\infty}\left(\Gamma_{1}, \mathbb{R}^{n}\right)$. In this paper we consider the functional

$$
B D(\Omega) \ni \mathbf{u} \mapsto \lambda F(\mathbf{u})+G_{j}(\varepsilon(\mathbf{u}))
$$

where

$$
\lambda F(\mathbf{u}) \equiv-\lambda L(\mathbf{u})+I_{C_{a}\left(\mathbf{u}^{0}\right)}(\mathbf{u}), \quad L(\mathbf{u}) \equiv \int_{\Omega} \mathbf{f} \cdot \mathbf{u} d x+\int_{\Gamma_{1}} \mathbf{g} \cdot \gamma_{B}(\mathbf{u}) d s,
$$

and define the subset $C_{a}\left(\mathbf{u}^{0}\right)$ of $B D(\Omega)$ by

$$
C_{a}\left(\mathbf{u}^{0}\right) \equiv\left\{\mathbf{u} \in B D(\Omega) \mid \gamma_{B}(\mathbf{u})_{\mid \Gamma_{0}}=\mathbf{u}^{0} \text { on } \Gamma_{0}, \mathbf{u}^{0} \in L^{1}\left(\Gamma_{0}, \mathbb{R}^{n}\right)\right\} .
$$

The functional $G_{j}: \mathbb{M}_{b}\left(\Omega, \mathbf{E}_{s}^{n}\right) \rightarrow \mathbb{R} \cup\{+\infty\}$ is given by

$$
G_{j}(\boldsymbol{\mu}) \equiv \begin{cases}\int_{\Omega} j(x, \boldsymbol{\mu}) d x & \text { if } \boldsymbol{\mu} \in L^{1}\left(\Omega, \mathbf{E}_{s}^{n}\right), \text { i.e. } \boldsymbol{\mu} \text { is absolutely } \\ & \text { continuous with respect to } d x \\ +\infty & \text { otherwise. }\end{cases}
$$

The expression (3.8) describes the total elastic-perfectly plastic energy of a body occupying the given subset $\Omega$ of $\mathbb{R}^{n}$. This body is subjected to volume forces $\mathbf{f} \in L^{n}\left(\Omega, \mathbb{R}^{n}\right)$ and boundary forces $\mathbf{g} \in L^{\infty}\left(\Gamma_{1}, \mathbb{R}^{n}\right)$. The constant $\lambda \geq 0, \lambda<\infty$ is the load multiplier (see [23, Chap. 1, Sec. 4]). The set $C_{a}\left(\mathbf{u}^{0}\right)$ consists of the kinematically admissible displacement fields for the body clamped on $\Gamma_{0}$ (see [6] and [23]).

Proposition 4 (see [23, p. 255]). If $\mathbf{u} \in B D\left(\Omega_{1}\right)$, then

$$
\varepsilon(\mathbf{u})=\varepsilon(\mathbf{u})_{\mid \Omega}+\varepsilon(\mathbf{u})_{\mid \Omega_{1}-\bar{\Omega}}+\left(\gamma_{B}^{O}(\mathbf{u})-\gamma_{B}^{I}(\mathbf{u})\right) \otimes_{s} \boldsymbol{\nu} d s,
$$

where the inside trace $\boldsymbol{\gamma}_{B}^{I}: B D(\Omega) \rightarrow L^{1}\left(\operatorname{Fr} \Omega, \mathbb{R}^{n}\right)$ and outside trace $\gamma_{B}^{O}$ : $B D\left(\Omega_{1}-\bar{\Omega}\right) \rightarrow L^{1}\left(\operatorname{Fr} \Omega, \mathbb{R}^{n}\right)$ are given by the formulae $\gamma_{B}^{I}(\mathbf{u})=\mathbf{u}_{\mid \operatorname{Fr}} \Omega$ for $\mathbf{u} \in B D(\Omega) \cap C\left(\bar{\Omega}, \mathbb{R}^{n}\right)$, and $\boldsymbol{\gamma}_{B}^{O}(\mathbf{u})=\mathbf{u}_{\mid \operatorname{Fr} \Omega}$ for $\mathbf{u} \in B D\left(\Omega_{1}-\bar{\Omega}\right) \cap$ 
$C\left(\Omega_{1}-\Omega, \mathbb{R}^{n}\right)$, respectively, and where $\otimes_{s}$ denotes the symmetric tensor product: $\left(\mathbf{p} \otimes_{s} \boldsymbol{\nu}\right)_{i j} \equiv\left(p_{i} \nu_{j}+p_{j} \nu_{i}\right) / 2$.

Definition 2 (see [16]). A Borel set $\mathcal{C} \subseteq \mathbb{R}^{n}$ is called a Caccioppoli set if $\sup \left\{\int_{\mathcal{C}} \operatorname{div} \widetilde{f} d x \mid \widetilde{f} \in C_{0}^{1}\left(\Omega_{2}, \mathbb{R}^{n}\right),\|\widetilde{f}(x)\|_{\mathbb{R}^{n}} \leq 1 \forall x \in \Omega_{2}\right\}<\infty$ for all bounded open subsets $\Omega_{2}$ of $\mathbb{R}^{n}$.

Remark 2. For every $\boldsymbol{\sigma} \in W^{n}\left(\Omega_{1}\right.$, div $)$ and $\mathbf{u} \in B D\left(\Omega_{1}\right)$ the distribution $\boldsymbol{\sigma}: \varepsilon(\mathbf{u})$ is a regular measure on $\Omega_{1}$. Then there exist sequences $\left\{\Omega_{c}^{k}\right\}_{k \in \mathbb{N}}$ and $\left\{\Omega_{0}^{k}\right\}_{k \in \mathbb{N}}$ of subsets of $\Omega_{1}$ such that

$$
\begin{gathered}
\operatorname{cl} \Omega_{c}^{k}=\Omega_{c}^{k} \subset \operatorname{Fr} \Omega \subset \Omega_{0}^{k}=\operatorname{int} \Omega_{0}^{k}, \quad \forall k \in \mathbb{N}, \\
\text { if } k_{1}<k_{2} \text { then } \Omega_{c}^{k_{1}} \subset \Omega_{c}^{k_{2}} \subset \Omega_{0}^{k_{2}} \subset \Omega_{0}^{k_{1}}, \\
|\boldsymbol{\sigma}: \boldsymbol{\varepsilon}(\mathbf{u})|\left(\Omega_{0}^{k}-\Omega_{c}^{k}\right)<1 / k, \quad \forall k \in \mathbb{N} .
\end{gathered}
$$

Moreover, by Urysohn's Lemma [13, Theorem 1.5.10], for every $k \in \mathbb{N}$, there exists a continuous function $\psi_{k}: \Omega_{1} \rightarrow[0,1]$ such that $\psi_{k}(x)=1$ for $x \in \Omega_{c}^{k}$ and $\psi_{k}(x)=0$ for $x \in \Omega_{1}-\Omega_{0}^{k}$. Then for every $\varphi \in C_{c}\left(\Omega_{1}\right)$ we have $\int_{\text {Fr } \Omega} \varphi \boldsymbol{\sigma}: \varepsilon(\mathbf{u})=\lim _{k \rightarrow \infty} \int_{\Omega_{1}} \psi_{k} \varphi \boldsymbol{\sigma}: \varepsilon(\mathbf{u})$ (cf. [2, Theorem 3.1]).

Lemma 5. If there exists a closed Caccioppoli set $\mathcal{C} \subset \Omega_{1}(\mathcal{C}=\operatorname{cl} \operatorname{int} \mathcal{C})$ such that $\Gamma_{2}=\operatorname{Fr} \Omega \cap \mathcal{C}$, with $d s(\operatorname{Fr} \Omega \cap \operatorname{Fr} \mathcal{C})=0$, then for all $\mathbf{u} \in B D\left(\Omega_{1}\right)$ and all $\boldsymbol{\sigma} \in W^{n}\left(\Omega_{1}\right.$, div $)$,

$$
\int_{\Gamma_{2}} \boldsymbol{\beta}_{B}\left(\boldsymbol{\sigma}_{\mid \Omega}\right) \cdot\left(\boldsymbol{\gamma}_{B}^{O}(\mathbf{u})-\boldsymbol{\gamma}_{B}^{I}(\mathbf{u})\right) d s=\int_{\Gamma_{2}} \boldsymbol{\sigma}:\left[\left(\boldsymbol{\gamma}_{B}^{O}(\mathbf{u})-\gamma_{B}^{I}(\mathbf{u})\right) \otimes_{s} \boldsymbol{\nu}\right] d s
$$

where we denote $\boldsymbol{\sigma}: \boldsymbol{\varepsilon}(\mathbf{u})_{\mid \operatorname{Fr} \Omega}$ by $\boldsymbol{\sigma}:\left[\left(\boldsymbol{\gamma}_{B}^{O}(\mathbf{u})-\boldsymbol{\gamma}_{B}^{I}(\mathbf{u})\right) \otimes_{s} \boldsymbol{\nu}\right] d s$.

Proof. Step 1. We prove that $\boldsymbol{\beta}_{B}\left(\boldsymbol{\sigma}_{\mid \Omega}\right)=-\boldsymbol{\beta}_{B}\left(\boldsymbol{\sigma}_{\mid \Omega_{1}-\bar{\Omega}}\right)$ on $\operatorname{Fr} \Omega$ for every $\boldsymbol{\sigma} \in W^{n}\left(\Omega_{1}\right.$, div $)$. Indeed, for every $\widehat{\mathbf{f}} \in L^{1}\left(\operatorname{Fr} \Omega, \mathbb{R}^{n}\right)$ there exist $\mathbf{u}_{1} \in$ $L D(\Omega)$ and $\mathbf{u}_{2} \in L D\left(\Omega_{1}-\bar{\Omega}\right)$ such that $\gamma_{B}\left(\mathbf{u}_{1}\right)=\gamma_{B}\left(\mathbf{u}_{2}\right)=\widehat{\mathbf{f}}$ (cf. [23, Chapter 2, Theorem 1.1]). By (2.11) and [23, Chapter 2, Lemma 2.2] we get

$$
\begin{aligned}
\int_{\Omega \cup\left(\Omega_{1}-\bar{\Omega}\right)}[\boldsymbol{\sigma}: \boldsymbol{\varepsilon}(\mathbf{u}) & +(\operatorname{div} \boldsymbol{\sigma}) \cdot \mathbf{u}] d x \\
& -\int_{\operatorname{Fr} \Omega}\left[\boldsymbol{\beta}_{B}\left(\boldsymbol{\sigma}_{\mid \Omega_{1}-\bar{\Omega}}\right)+\boldsymbol{\beta}_{B}\left(\boldsymbol{\sigma}_{\mid \Omega}\right)\right] \cdot \boldsymbol{\gamma}_{B}\left(\mathbf{u}_{\mid \Omega}\right) d s \\
= & \int_{\Omega_{1}} \boldsymbol{\sigma}: \varepsilon(\mathbf{u}) d x+\int_{\Omega_{1}}(\operatorname{div} \boldsymbol{\sigma}) \cdot \mathbf{u} d x
\end{aligned}
$$

for every $(\mathbf{u}, \boldsymbol{\sigma}) \in L D\left(\Omega_{1}\right) \times W^{n}\left(\Omega_{1}\right.$, div $)$. Therefore, for every $(\widehat{\mathbf{f}}, \boldsymbol{\sigma}) \in$ $L^{1}\left(\operatorname{Fr} \Omega, \mathbb{R}^{n}\right) \times W^{n}\left(\Omega_{1}, \operatorname{div}\right), \int_{\operatorname{Fr} \Omega}\left[\boldsymbol{\beta}_{B}\left(\boldsymbol{\sigma}_{\mid \Omega_{1}-\bar{\Omega}}\right)+\boldsymbol{\beta}_{B}\left(\boldsymbol{\sigma}_{\mid \Omega}\right)\right] \cdot \widehat{\mathbf{f}} d s=0$. 
Step 2. Let $\mathbf{u}_{1} \in B D\left(\Omega_{1}\right)$ and $\mathbf{u}_{1 \mid \Omega_{1}-\bar{\Omega}}=\mathbf{0}$. By Proposition 4 and (2.11),

$$
\int_{\Omega} \boldsymbol{\sigma}: \varepsilon\left(\mathbf{u}_{1}\right)+\int_{\Omega}(\operatorname{div} \boldsymbol{\sigma}) \cdot \mathbf{u}_{1} d x=\int_{\operatorname{Fr} \Omega} \boldsymbol{\sigma}:\left(\boldsymbol{\gamma}_{B}^{I}\left(\mathbf{u}_{1}\right) \otimes_{s} \boldsymbol{\nu}\right) d s .
$$

Replacing $\Omega$ by $\Omega_{1}-\bar{\Omega}$, we get

$$
\int_{\operatorname{Fr} \Omega} \boldsymbol{\beta}_{B}\left(\boldsymbol{\sigma}_{\mid \Omega}\right) \cdot\left(\boldsymbol{\gamma}_{B}^{O}(\mathbf{u})-\boldsymbol{\gamma}_{B}^{I}(\mathbf{u})\right) d s=\int_{\operatorname{Fr} \Omega} \boldsymbol{\sigma}:\left[\left(\boldsymbol{\gamma}_{B}^{O}(\mathbf{u})-\boldsymbol{\gamma}_{B}^{I}(\mathbf{u})\right) \otimes_{s} \boldsymbol{\nu}\right] d s
$$

for $\mathbf{u} \in B D\left(\Omega_{1}\right)$. Let $\mathcal{X}_{\mathcal{C}}(x)=1$ if $x \in \mathcal{C}$ and $\mathcal{X}_{\mathcal{C}}(x)=0$ otherwise. Then, for every $\mathbf{u} \in B D\left(\Omega_{1}\right), \mathcal{X}_{\mathcal{C}} \mathbf{u} \in B D\left(\Omega_{1}\right)$ and we get

$$
\begin{aligned}
\int_{\mathcal{C} \cap \mathrm{Fr} \Omega} \boldsymbol{\beta}_{B}(\boldsymbol{\sigma})\left(\boldsymbol{\gamma}_{B}^{O}(\mathbf{u})-\boldsymbol{\gamma}_{B}^{I}(\mathbf{u})\right) d s & =\int_{\operatorname{Fr} \Omega} \boldsymbol{\beta}_{B}(\boldsymbol{\sigma})\left(\boldsymbol{\gamma}_{B}^{O}\left(\mathcal{X}_{\mathcal{C}} \mathbf{u}\right)-\gamma_{B}^{I}\left(\mathcal{X}_{\mathcal{C}} \mathbf{u}\right)\right) d s \\
& =\int_{\Gamma_{2}} \boldsymbol{\sigma}:\left[\left(\boldsymbol{\gamma}_{B}^{O}(\mathbf{u})-\gamma_{B}^{I}(\mathbf{u})\right) \otimes_{s} \boldsymbol{\nu}\right] d s .
\end{aligned}
$$

Assumption 4. Let $\Gamma_{1}=\operatorname{Fr} \Omega \cap \mathcal{C}$, where $\mathcal{C}=\operatorname{clint} \mathcal{C} \subset \Omega_{1}$ is a closed Caccioppoli set and $d s(\operatorname{Fr} \Omega \cap \operatorname{Fr} \mathcal{C})=0$.

Let $\boldsymbol{\mu} \in \mathbb{M}_{b}\left(\Omega, \mathbf{E}_{s}^{n}\right)$. We recall that $|\boldsymbol{\mu}|$ is the total variation measure associated with $\boldsymbol{\mu}$, i.e. for every $\boldsymbol{\mu}$-measurable subset $\widetilde{\Omega}$ of $\Omega$ we have $|\boldsymbol{\mu}|(\widetilde{\Omega})=\sup \left\{\int_{\widetilde{\Omega}} \boldsymbol{\varphi}: \boldsymbol{\mu} \mid \boldsymbol{\varphi} \in C_{0}\left(\Omega, \mathbf{E}_{s}^{n}\right), \max _{i, j}\left(\left\|\varphi_{i j}\right\|_{C(\Omega)}\right) \leq 1\right\}$. Then $\|\boldsymbol{\mu}\|_{\mathbb{M}_{b}(\Omega)}=\int_{\Omega}|\boldsymbol{\mu}|$. The density of $\boldsymbol{\mu}$ with respect to $|\boldsymbol{\mu}|$ will be denoted by $d \boldsymbol{\mu} / d|\boldsymbol{\mu}|$. Let $\boldsymbol{\mu}=\boldsymbol{\mu}_{a}(x) d x+\boldsymbol{\mu}_{s}$ be the Lebesgue decomposition of $\boldsymbol{\mu}$ into absolutely continuous and singular parts with respect to $d x$.

4. The scheme of duality in Hencky plasticity. In this section we define the duality between the displacement formulation and the stress formulation of the variational problem in Hencky plasticity (cf. [12, Chapter 3]). We prove (similarly to [25]) the existence theorem for the stress problem (see Theorem 7) for an elastic-perfectly plastic solid, made of a non-homogeneous Hencky material, where the following condition is fulfilled:

$$
\exists r_{2}>0, \forall x \in \bar{\Omega} \quad \mathcal{K}(x) \subset B_{\mathbf{E}_{s}^{n}}\left(0, r_{2}\right) .
$$

Let

$$
V \equiv\left[L D(\Omega),\|\cdot\|_{L D}\right], \quad Y \equiv\left[L^{1}\left(\Omega, \mathbf{E}_{s}^{n}\right),\|\cdot\|_{L^{1}\left(\Omega, \mathbf{E}_{s}^{n}\right)}\right]
$$

(cf. [12, Chapter 3]). Moreover, let

$$
V^{*}=L D^{*}(\Omega)=\left[L D(\Omega),\|\cdot\|_{L D}\right]^{*}, \quad Y^{*}=\left[L^{\infty}\left(\Omega, \mathbf{E}_{s}^{n}\right), \sigma\left(L^{\infty}, L^{1}\right)\right] .
$$

The linear operator $\varepsilon: L D(\Omega) \rightarrow L^{1}\left(\Omega, \mathbf{E}_{s}^{n}\right)=Y$ is continuous (cf. (2.1)). Below, the following functional is considered:

$$
L D(\Omega) \ni \mathbf{u} \mapsto \lambda F(\mathbf{u})+G_{j}(\varepsilon(\mathbf{u})) .
$$


Let $\gamma_{B}\left(\mathbf{u}_{0}\right)=\mathbf{u}^{0}$ on $\Gamma_{0}$ where $\mathbf{u}_{0} \in L D(\Omega)$ (see [23, Chapter 2, Theorem $1.1])$.

Lemma 6 (cf. [25] and [23, Chapter 1, Lemma 2.2]). The dual problem of

$$
\left(P_{\lambda}\right) \quad \text { find } \inf \left\{\lambda F(\mathbf{u})+G_{j}(\varepsilon(\mathbf{u})) \mid \mathbf{u} \in L D(\Omega)\right\}
$$

is

$$
\left(P_{\lambda}^{*}\right) \quad \text { find } \sup \left\{-(\lambda F)^{*}\left(-\varepsilon^{*}(\boldsymbol{\sigma})\right)-G_{j}^{*}(\boldsymbol{\sigma}) \mid \boldsymbol{\sigma} \in L^{\infty}\left(\Omega, \mathbf{E}_{s}^{n}\right)\right\},
$$

where

$$
(\lambda F)^{*}\left(-\varepsilon^{*}(\boldsymbol{\sigma})\right)= \begin{cases}-\int_{\Gamma_{0}} \boldsymbol{\beta}_{B}(\boldsymbol{\sigma}) \cdot \mathbf{u}^{0} d s & \text { if } \operatorname{div} \boldsymbol{\sigma}=-\lambda \mathbf{f} \text { in } \Omega \\ +\infty & \text { and } \boldsymbol{\beta}_{B}(\boldsymbol{\sigma})=\lambda \mathbf{g} \text { on } \Gamma_{1}, \\ & \text { otherwise }\end{cases}
$$

and

$$
G_{j}^{*}(\boldsymbol{\sigma})=\int_{\Omega} j^{*}(x, \boldsymbol{\sigma}) d x .
$$

The trace $\boldsymbol{\beta}_{B}(\boldsymbol{\sigma}) \in L^{\infty}\left(\operatorname{Fr} \Omega, \mathbb{R}^{n}\right)$ exists (cf. Theorem 3$)$. Moreover, $\boldsymbol{\varepsilon}^{*}(\boldsymbol{\sigma})=$ $\left(\operatorname{div} \boldsymbol{\sigma}, \boldsymbol{\beta}_{B}(\boldsymbol{\sigma})\right)$, where the bilinear pairing between $V$ and $\boldsymbol{\varepsilon}^{*}\left(Y^{*}\right)$ is given by

$$
\left\langle\mathbf{u}, \varepsilon^{*}(\boldsymbol{\sigma})\right\rangle_{V \times \varepsilon^{*}\left(Y^{*}\right)}=-\int_{\Omega}(\operatorname{div} \boldsymbol{\sigma}) \mathbf{u} d x+\int_{\operatorname{Fr} \Omega} \boldsymbol{\beta}_{B}(\boldsymbol{\sigma}) \boldsymbol{\gamma}_{B}(\mathbf{u}) d s .
$$

Proof. (i) First we prove (4.8). Because of (3.3), the function $j$ is a convex normal integrand. By [21, Theorem 3A and Proposition $2 \mathrm{M}]$ we get (4.8), since $L^{1}\left(\Omega, \mathbf{E}_{s}^{n}\right)$ is a decomposable space and $j(x, \mathbf{0}) \leq 0$ for $d x$-a.e. $x \in \Omega$ ( $j^{*}$ is a non-negative function).

(ii) We apply Lemma 2.1 of [23, Chapter 1] with $v_{0}=\mathbf{u}_{0} \in \widetilde{V}=L D(\Omega)$,

$$
\left\langle v_{0}^{*}, \mathbf{u}\right\rangle_{L D^{*} \times L D}=-\lambda\left(\int_{\Omega} \mathbf{f} \cdot \mathbf{u} d x+\int_{\Gamma_{1}} \mathbf{g} \cdot \gamma_{B}(\mathbf{u}) d s\right), \quad \forall \mathbf{u} \in L D(\Omega),
$$

and where $\mathcal{B}$ is the set of $\mathbf{u}$ in $L D(\Omega)$ such that $\boldsymbol{\gamma}_{B}(\mathbf{u})$ vanishes on $\Gamma_{0}$. We deduce from this lemma that $\left(\lambda F_{1}\right)^{*}\left(-\boldsymbol{\varepsilon}^{*}(\boldsymbol{\sigma})\right)$ is equal to

$$
Q_{\mathbf{u}_{0}}(\boldsymbol{\sigma}) \equiv\left\langle-\varepsilon^{*}(\boldsymbol{\sigma}), \mathbf{u}_{0}\right\rangle_{L D^{*} \times L D}+\lambda\left(\int_{\Omega} \mathbf{f} \cdot \mathbf{u}_{0} d x+\int_{\Gamma_{1}} \mathbf{g} \cdot \gamma_{B}\left(\mathbf{u}_{0}\right) d s\right)
$$

if $Q_{\mathbf{u}}(\boldsymbol{\sigma})=0$ for every $\mathbf{u} \in \mathcal{B}$, and to $+\infty$ if $Q_{\mathbf{u}_{1}}(\boldsymbol{\sigma}) \neq 0$ for some $\mathbf{u}_{1} \in \mathcal{B}$. Writing $Q_{\mathbf{u}}(\boldsymbol{\sigma})=0$ with $\mathbf{u}$ replaced by $\widehat{\mathbf{u}} \in L D_{0} \equiv\left\{\mathbf{u} \in L D(\Omega) \mid \boldsymbol{\gamma}_{B}(\mathbf{u})=\mathbf{0}\right.$ on $\operatorname{Fr} \Omega$ \}, we see that

$$
\begin{aligned}
& \langle-\boldsymbol{\sigma}, \boldsymbol{\varepsilon}(\widehat{\mathbf{u}})\rangle_{L^{\infty} \times L^{1}}+\lambda \int_{\Omega} \mathbf{f} \cdot \widehat{\mathbf{u}} d x=0, \\
& \left\langle-\varepsilon^{*}(\boldsymbol{\sigma}), \widehat{\mathbf{u}}\right\rangle_{L D^{*} \times L D}+\lambda \int_{\Omega} \mathbf{f} \cdot \widehat{\mathbf{u}} d x=\left\langle\lambda \mathbf{f}-\boldsymbol{\varepsilon}^{*}(\boldsymbol{\sigma}), \widehat{\mathbf{u}}\right\rangle_{L D^{*} \times L D}=0
\end{aligned}
$$


for every $\widehat{\mathbf{u}} \in L D_{0}(\Omega)$. By (2.11) and (4.12) we obtain $\int_{\Omega}(\lambda \mathbf{f}+\operatorname{div} \boldsymbol{\sigma})$. $\widehat{\mathbf{u}} d x=0$ for every $\widehat{\mathbf{u}} \in L D_{0}(\Omega)$, or in other words $\lambda \mathbf{f}=-\operatorname{div} \boldsymbol{\sigma}$ in the sense of distributions on $\Omega$. The trace $\boldsymbol{\beta}_{B}(\boldsymbol{\sigma})$ on $\operatorname{Fr} \Omega$ exists, because $\operatorname{div} \boldsymbol{\sigma}=$ $-\lambda \mathbf{f} \in L^{n}\left(\Omega, \mathbb{R}^{n}\right)$ (see Theorem 3). In the case when $Q_{\mathbf{u}}(\boldsymbol{\sigma})=0$ for every $\mathbf{u} \in \mathcal{B}$, by (2.11), we have

$$
0=\int_{\Gamma_{1}}\left(\lambda \mathbf{g}-\boldsymbol{\beta}_{B}(\boldsymbol{\sigma})\right) \cdot \gamma_{B}(\mathbf{u}) d s, \quad \forall \mathbf{u} \in \mathcal{B},
$$

because $\operatorname{div} \boldsymbol{\sigma}=-\lambda \mathbf{f}$ and $\gamma_{B}(\mathbf{u})=\mathbf{0}$ on $\Gamma_{0}$ for every $\mathbf{u} \in \mathcal{B}$. The trace $\boldsymbol{\gamma}_{B}$ is a function onto $L^{1}\left(\operatorname{Fr} \Omega, \mathbb{R}^{n}\right)$, hence $\boldsymbol{\beta}_{B}(\boldsymbol{\sigma})=\lambda \mathbf{g}$ on $\Gamma_{1}$. By (4.11) and (2.11) we obtain

$$
\begin{aligned}
& \left(\lambda F_{1}\right)^{*}\left(-\boldsymbol{\varepsilon}^{*}(\boldsymbol{\sigma})\right) \\
= & \left\langle-\boldsymbol{\sigma}, \boldsymbol{\varepsilon}\left(\mathbf{u}_{0}\right)\right\rangle_{L^{\infty} \times L^{1}}+\lambda\left(\int_{\Omega} \mathbf{f} \cdot \mathbf{u}_{0} d x+\int_{\Gamma_{1}} \mathbf{g} \cdot \boldsymbol{\gamma}_{B}\left(\mathbf{u}_{0}\right) d s\right) \\
= & \int_{\Omega}(\lambda \mathbf{f}+\operatorname{div} \boldsymbol{\sigma}) \cdot \mathbf{u}_{0} d x-\int_{\operatorname{Fr} \Omega} \boldsymbol{\beta}_{B}(\boldsymbol{\sigma}) \cdot \gamma_{B}\left(\mathbf{u}_{0}\right) d s+\lambda \int_{\Gamma_{1}} \mathbf{g} \cdot \boldsymbol{\gamma}_{B}\left(\mathbf{u}_{0}\right) d s \\
= & -\int_{\Gamma_{0}} \boldsymbol{\beta}_{B}(\boldsymbol{\sigma}) \cdot \gamma_{B}\left(\mathbf{u}_{0}\right) d s .
\end{aligned}
$$

Theorem 7 (see [25] and [23]). Suppose $\inf \left(P_{\lambda}\right)$ is finite. Moreover, assume that inclusion (4.1) holds. Then $\inf \left(P_{\lambda}\right)=\sup \left(P_{\lambda}^{*}\right)$ and $\left(P_{\lambda}^{*}\right)$ has at least one solution $\sigma_{0} \in W^{n}(\Omega$, div $)$, where $\left(P_{\lambda}^{*}\right)$ is defined by (4.6)-(4.8).

Proof. The function

$$
L^{1}\left(\Omega, \mathbf{E}_{s}^{n}\right) \ni \mathbf{p} \mapsto G_{1, j}(\varepsilon(\mathbf{u})+\mathbf{p}) \in \mathbb{R} \cup\{+\infty\}
$$

is l.s.c. in the topology $\sigma\left(L^{1}\left(\Omega, \mathbf{E}_{s}^{n}\right), L^{\infty}\left(\Omega, \mathbf{E}_{s}^{n}\right)\right)$, where $\mathbf{u} \in L D(\Omega)$. Indeed, by (4.8) and [21, Theorem 3A and Proposition $2 \mathrm{M}$ ] we get

$$
\begin{aligned}
G_{j}^{* *}(\mathbf{p}) & \equiv \sup \left\{\int_{\Omega} \mathbf{p}: \boldsymbol{\sigma} d x-\int_{\Omega} j^{*}(x, \boldsymbol{\sigma}) d x \mid \boldsymbol{\sigma} \in L^{\infty}\left(\Omega, \mathbf{E}_{s}^{n}\right)\right\} \\
& =\int_{\Omega} j(x, \mathbf{p}) d x, \quad \forall \mathbf{p} \in L^{1}\left(\Omega, \mathbf{E}_{s}^{n}\right),
\end{aligned}
$$

since $j^{* *}=j$ (cf. (3.11)). By the Mazur Lemma the function (4.16) is l.s.c. in the norm $\|\cdot\|_{L^{1}}$, because the epigraph of (4.16) is closed in the norm $\mathbb{R} \times$ $L^{1}\left(\Omega, \mathbf{E}_{s}^{n}\right) \ni(z, \mathbf{p}) \mapsto|z|+\|\mathbf{p}\|_{L^{1}\left(\Omega, \mathbf{E}_{s}^{n}\right)}$. By (4.1) we have $j(x, \mathbf{w})=j^{* *}(x, \mathbf{w})$ $\leq \bar{c}_{n} r_{2}\|\mathbf{w}\|_{\mathbf{E}_{s}^{n}}$ for every $\mathbf{w} \in \mathbf{E}_{s}^{n}$ and $d x$-a.e. $x \in \Omega$, where $\bar{c}_{n}>0$ depends only on $n$. Thus $\operatorname{dom} G_{j}=L^{1}\left(\Omega, \mathbf{E}_{s}^{n}\right)$. Because of [12, Chapter 1 , Corollary $2.5]$, the function (4.16) is continuous on the whole space $\left[L^{1}\left(\Omega, \mathbf{E}_{s}^{n}\right),\|\cdot\|_{L^{1}}\right]$. By [12, Chapter 3, Theorem 4.1] the proof is complete. 
5. The scheme of duality for the relaxed functional. In this section we define the functional of energy, where the work of boundary forces is relaxed. We find the dual problem and show the existence theorem.

Let the condition (4.1) be satisfied. Moreover, let

$$
\begin{aligned}
\lambda F_{r}(\mathbf{u}) \equiv & -\lambda\left(\int_{\Omega} \mathbf{f} \cdot \mathbf{u} d x+\int_{\Gamma_{1}} \mathbf{g} \cdot \boldsymbol{\gamma}_{B}(\mathbf{u}) d s\right) \\
& +\int_{\Gamma_{1}} r\left\|\boldsymbol{\gamma}_{B}(\mathbf{u})\right\|_{\mathbb{R}^{n}}^{E} d s+I_{C_{a}\left(\mathbf{u}^{0}\right)}(\mathbf{u})
\end{aligned}
$$

for every $\mathbf{u} \in L D(\Omega)$, where $\|\cdot\|_{\mathbb{R}^{n}}^{E}$ is the Euclidean norm in $\mathbb{R}^{n}$.

Definition 3 (cf. [8]). A subset $H_{0}$ of $\mathcal{L}^{0}\left(\bar{\Omega}, \mathbb{R}^{m}\right)_{\mu}$ is said to be $P C U$ stable if for any continuous partition of unity $\left(\alpha_{0}, \ldots, \alpha_{d}\right)$ such that $\alpha_{0}, \ldots$, $\alpha_{d} \in C^{\infty}(\bar{\Omega}, \mathbb{R})$, and any $\mathbf{z}_{0}, \ldots, \mathbf{z}_{d} \in H_{0}$, the sum $\sum_{i=0}^{d} \alpha_{i} \mathbf{z}_{i}$ is in $H_{0}$.

Lemma 8. The dual problem to the relaxed formula

$$
\left(P_{\lambda, r}\right) \quad \text { find } \inf \left\{\lambda F_{r}(\mathbf{u})+G_{j}(\varepsilon(\mathbf{u})) \mid \mathbf{u} \in L D(\Omega)\right\},
$$

is

$$
\left(P_{\lambda, r}^{*}\right) \quad \text { find } \sup \left\{-\left(\lambda F_{r}\right)^{*}\left(-\varepsilon^{*}(\boldsymbol{\sigma})\right)-G_{j}^{*}(\boldsymbol{\sigma}) \mid \boldsymbol{\sigma} \in L^{\infty}\left(\Omega, \mathbf{E}_{s}^{n}\right)\right\},
$$

where

$$
\left(\lambda F_{r}\right)^{*}\left(-\boldsymbol{\varepsilon}^{*}(\boldsymbol{\sigma})\right)= \begin{cases}\int_{\Gamma_{1}} I_{B^{E}(0, r)}\left(\lambda \mathbf{g}-\boldsymbol{\beta}_{B}(\boldsymbol{\sigma})\right) & \text { ds }-\int_{\Gamma_{0}} \boldsymbol{\beta}_{B}(\boldsymbol{\sigma}) \cdot \mathbf{u}^{0} d s \\ +\infty & \text { if } \operatorname{div} \boldsymbol{\sigma}=-\lambda \mathbf{f} \text { in } \Omega \\ \text { otherwise }\end{cases}
$$

and $G_{j}^{*}$ is given in (4.8). Here $B^{E}(0, r)$ is the closed ball in the space $\mathbb{R}^{n}$, endowed with Euclidean norm $\|\cdot\|_{\mathbb{R}^{n}}^{E}$.

Proof. In view of the proof of Lemma 6, it suffices to show (5.4) for $\boldsymbol{\sigma} \in Y^{*}$. Since $L D(\Omega)$ is $P C U$-stable, by [8, Theorem 1] we obtain

$$
\begin{aligned}
(\lambda F)^{*}\left(-\varepsilon^{*}(\boldsymbol{\sigma})\right) \equiv \sup _{\mathbf{u} \in V}\left\{\left\langle\mathbf{u},-\boldsymbol{\varepsilon}^{*}(\boldsymbol{\sigma})\right\rangle_{V \times \boldsymbol{\varepsilon}^{*}\left(Y^{*}\right)}-\lambda F_{r}(\mathbf{u})\right\} \\
=\sup _{\mathbf{u} \in V}\left\{\int_{\Omega}(\operatorname{div} \boldsymbol{\sigma}) \mathbf{u} d x-\int_{\operatorname{Fr} \Omega} \boldsymbol{\beta}_{B}(\boldsymbol{\sigma}) \boldsymbol{\gamma}_{B}(\mathbf{u}) d s+\lambda\left(\int_{\Omega} \mathbf{f} \cdot \mathbf{u} d x\right.\right. \\
\left.\left.\quad+\int_{\Gamma_{1}} \mathbf{g} \cdot \boldsymbol{\gamma}_{B}(\mathbf{u}) d s\right)-\int_{\Gamma_{1}} r\left\|\boldsymbol{\gamma}_{B}(\mathbf{u})\right\|_{\mathbb{R}^{n}}^{E} d s-I_{C_{a}\left(\mathbf{u}^{0}\right)}(\mathbf{u})\right\} \\
=\sup _{\mathbf{u} \in V}\left\{\int_{\Omega}[\operatorname{div} \boldsymbol{\sigma}+\lambda \mathbf{f}] \mathbf{u} d x+\int_{\Gamma_{1}}\left(\lambda \mathbf{g}-\boldsymbol{\beta}_{B}(\boldsymbol{\sigma})\right) \boldsymbol{\gamma}_{B}(\mathbf{u}) d s\right.
\end{aligned}
$$




$$
\begin{aligned}
& \left.-\int_{\Gamma_{1}} r\left\|\gamma_{B}(\mathbf{u})\right\|_{\mathbb{R}^{n}}^{E} d s-\int_{\Gamma_{0}}\left[\boldsymbol{\beta}_{B}(\boldsymbol{\sigma}) \cdot \boldsymbol{\gamma}_{B}(\mathbf{u})-I_{\left\{\mathbf{z} \in \mathbb{R}^{n} \mid \mathbf{z}=\mathbf{u}^{0}\right\}}\left(\boldsymbol{\gamma}_{B}(\mathbf{u})\right)(x)\right] d s\right\} \\
= & \int_{\Omega} I_{\left\{\mathbf{z} \in \mathbb{R}^{n} \mid \mathbf{z}=-\mathbf{f}\right\}}(\operatorname{div} \boldsymbol{\sigma}) d x+\int_{\Gamma_{1}} I_{B^{E}(0, r)}\left(\lambda \mathbf{g}-\boldsymbol{\beta}_{B}(\boldsymbol{\sigma})\right) d s-\int_{\Gamma_{0}} \boldsymbol{\beta}_{B}(\boldsymbol{\sigma}) \cdot \mathbf{u}^{0} d s .
\end{aligned}
$$

TheOREM 9. Suppose $\inf \left(P_{\lambda, r}\right)$ is finite. Moreover, assume that inclusion (4.1) holds. Then $\inf \left(P_{\lambda, r}\right)=\sup \left(P_{\lambda, r}^{*}\right)$ and $\left(P_{\lambda, r}^{*}\right)$ has at least one solution $\boldsymbol{\sigma}_{0} \in W^{n}(\Omega, \operatorname{div})$, where $\left(P_{\lambda, r}^{*}\right)$ is defined by $(4.8),(5.3)$ and $(5.4)$.

Proof. Similar to that of Theorem 7.

6. Regularity of displacement solutions. In this section it is proved that the displacement solutions of the relaxed functional belong to the space $L D(\Omega)$ (cf. Theorem 21). In this section we assume that $\mathbf{u}^{0}=\mathbf{0}$ on $\Gamma_{0}$. Moreover, we do not assume that the set $\mathcal{K}(x)$ is bounded for any $x \in \bar{\Omega}$.

The functional $\left\lceil P_{\lambda, r}^{*}\right\rceil$ is defined by the expression

$$
W^{n}(\Omega, \operatorname{div}) \ni \boldsymbol{\sigma} \mapsto\left\lceil P_{\lambda, r}^{*}\right\rceil(\boldsymbol{\sigma})=-\left(\lambda F_{r}\right)^{*}\left(-\varepsilon^{*}(\boldsymbol{\sigma})\right)-G_{j}^{*}(\boldsymbol{\sigma}),
$$

where $\left(\lambda F_{r}\right)^{*}$ and $G_{j}^{*}$ are given by (5.4) and (4.8). We define

$$
\begin{aligned}
\mathbf{Y}^{1}(\bar{\Omega}) \equiv\left\{\mathbf{M} \in \mathbb{M}_{b}\left(\bar{\Omega}, \mathbf{E}_{s}^{n}\right) \mid \exists \mathbf{u}_{1} \in B D\left(\Omega_{1}\right),\right. \\
\left.\qquad\left(\mathbf{u}_{1}\right)_{\mid \bar{\Omega}}=\mathbf{M}, \mathbf{u}_{1 \mid \Omega_{1}-\bar{\Omega}}=\mathbf{0}\right\} .
\end{aligned}
$$

The bilinear pairing between $\mathbf{M} \in \mathbf{Y}^{1}(\bar{\Omega})$ and $\boldsymbol{\sigma} \in W^{n}(\Omega$, div) is introduced below. Let $\boldsymbol{\varepsilon}(\mathbf{u})=\mathbf{M}$, where $\mathbf{u} \in B D\left(\Omega_{1}\right)$ and $\mathbf{u}_{\mid \Omega_{1}-\bar{\Omega}}=\mathbf{0}$. Moreover, let $\boldsymbol{\sigma}_{1} \in W^{n}\left(\Omega_{1}\right.$, div) where $\boldsymbol{\sigma}_{1 \mid \Omega}=\boldsymbol{\sigma}$ (see Remark 1); then we define

$$
\begin{aligned}
\langle\mathbf{M}, \boldsymbol{\sigma}\rangle_{\mathbf{Y}^{1} \times W^{n}(\Omega, \operatorname{div})} & =\int_{\bar{\Omega}} \boldsymbol{\sigma}_{1}: \boldsymbol{\varepsilon}(\mathbf{u}) \\
& =\int_{\Omega} \boldsymbol{\sigma}: \boldsymbol{\varepsilon}(\mathbf{u})_{\mid \Omega}-\int_{\operatorname{Fr} \Omega} \boldsymbol{\beta}_{B}(\boldsymbol{\sigma}) \cdot \gamma_{B}^{I}(\mathbf{u}) d s
\end{aligned}
$$

(cf. formulae (2.10), (3.12) and (3.16)).

REMARK 3. The definition of spaces in duality requires that for every $\boldsymbol{\sigma} \in W^{n}(\Omega, \operatorname{div}), \boldsymbol{\sigma} \neq \mathbf{0}$, there exists $\mathbf{M}=\boldsymbol{\varepsilon}(\mathbf{u}) \in \mathbf{Y}^{1}(\bar{\Omega})$ such that

$$
\int_{\bar{\Omega}} \boldsymbol{\sigma}: \mathbf{M}=\int_{\Omega} \boldsymbol{\sigma}: \varepsilon(\mathbf{u})-\int_{\operatorname{Fr} \Omega} \boldsymbol{\beta}_{B}(\boldsymbol{\sigma}) \cdot \gamma_{B}^{I}(\mathbf{u}) d s \neq 0 .
$$

But for every $\boldsymbol{\sigma} \in W^{n}(\Omega, \operatorname{div})$ such that $\operatorname{div} \boldsymbol{\sigma}=\mathbf{0}$ in $\Omega$, and for every $\mathbf{M}=\varepsilon(\mathbf{u}) \in \mathbf{Y}^{1}(\bar{\Omega})$,

$$
\int_{\Omega} \boldsymbol{\sigma}: \varepsilon(\mathbf{u})-\int_{\operatorname{Fr} \Omega} \boldsymbol{\sigma}:\left(\gamma_{B}^{I}(\mathbf{u}) \otimes_{s} \boldsymbol{\nu}\right) d s=-\int_{\Omega}(\operatorname{div} \boldsymbol{\sigma}) \cdot \mathbf{u} d x=0
$$

(see (2.11) and (3.16)). Therefore the duality should be defined between the spaces $\mathbf{Y}^{1}(\bar{\Omega})$ and $W^{n}(\Omega, \operatorname{div}) /\left\{\boldsymbol{\sigma} \in C\left(\bar{\Omega}, \mathbf{E}_{s}^{n}\right) \mid \operatorname{div} \boldsymbol{\sigma}=\mathbf{0}\right\}$. To simplify the 
proofs, the previous definition, given by (2.9) and (6.3), is considered here. We do not get a contradiction, since we do not use the Hausdorff property of the topology $\sigma\left(W^{n}(\Omega, \operatorname{div}), \mathbf{Y}^{1}(\bar{\Omega})\right)$.

We define the subspace $C_{\operatorname{div}}\left(\bar{\Omega}, \mathbf{E}_{s}^{n}\right)$ of $W^{n}(\Omega, \operatorname{div})$ by

$$
C_{\text {div }}\left(\bar{\Omega}, \mathbf{E}_{s}^{n}\right) \equiv\left\{\boldsymbol{\sigma} \in C\left(\bar{\Omega}, \mathbf{E}_{s}^{n}\right) \mid \boldsymbol{\sigma}_{\mid \Omega} \in W^{n}(\Omega, \operatorname{div})\right\} .
$$

We say that a net $\left\{\mathbf{M}_{\delta}\right\}_{\delta \in D} \subset \mathbf{Y}^{1}(\bar{\Omega})$ is convergent to $\mathbf{M}_{0} \in \mathbf{Y}^{1}$ in the topology $\sigma\left(\mathbf{Y}^{1}(\bar{\Omega}), C_{\text {div }}\left(\bar{\Omega}, \mathbf{E}_{s}^{n}\right)\right)$ if $\left\langle\mathbf{M}_{\delta}-\mathbf{M}_{0}, \boldsymbol{\sigma}\right\rangle_{\mathbf{Y}^{1} \times W^{n}(\Omega, \text { div })} \rightarrow 0$ for all $\boldsymbol{\sigma} \in C_{\text {div }}\left(\bar{\Omega}, \mathbf{E}_{s}^{n}\right)$. Similarly, a net $\left\{\mathbf{M}_{\delta}\right\}_{\delta \in D} \subset \mathbf{Y}^{1}$ is convergent to $\mathbf{M}_{0}$ in $\sigma\left(\mathbf{Y}^{1}(\bar{\Omega}), W^{n}(\Omega, \operatorname{div})\right)$ if $\left\langle\mathbf{M}_{\delta}-\mathbf{M}_{0}, \boldsymbol{\sigma}\right\rangle_{\mathbf{Y}^{1} \times W^{n}(\Omega, \text { div })} \rightarrow 0$ for all $\boldsymbol{\sigma} \in$ $W^{n}(\Omega, \operatorname{div})$.

The space $B D(\Omega)$ is isomorphic to $\mathcal{A} \equiv\left\{\mathbf{u} \in B D\left(\Omega_{1}\right) \mid \mathbf{u}_{\mid \Omega_{1}-\bar{\Omega}}=\mathbf{0}\right\}$ (cf. Assumption 1). Moreover, $\mathcal{A}$ is isomorphic to $\mathbf{Y}^{1}(\bar{\Omega})$, and the isomorphism is given by the formula

$$
\left\{\mathbf{u} \in B D\left(\Omega_{1}\right) \mid \mathbf{u}_{\mid \Omega_{1}-\bar{\Omega}}=\mathbf{0}\right\}=\mathcal{A} \ni \mathbf{u} \mapsto \varepsilon(\mathbf{u})_{\mid \bar{\Omega}} \in \mathbf{Y}^{1}(\bar{\Omega}) .
$$

The Banach spaces $\left[B D(\Omega),\|\cdot\|_{B D}\right]$ and $\left[\mathbf{Y}^{1}(\bar{\Omega}),\|\cdot\|_{\mathbb{M}_{b}(\bar{\Omega})}\right]$ are isomorphic (cf. [6, Proposition 4.24]). Each closed ball $\mathrm{cl}_{\|\cdot\|}\left(B(0, r)\right.$ ) (in $\mathbf{Y}^{1}$ ) is compact in the topology $\sigma\left(\mathbf{Y}^{1}(\bar{\Omega}), C_{\operatorname{div}}\left(\bar{\Omega}, \mathbf{E}_{s}^{n}\right)\right)$, where $\mathrm{cl}_{\|\cdot\|}$ denotes the closure in the norm of $B D(\Omega)$ (see $[6$, Proposition 4.23$]$ ). The space $\left[\mathrm{cl}_{\|\cdot\|_{B D}}\left(B_{B D}\left(0, r_{2}\right)\right)\right.$, weak* $B D(\Omega)$ topology] is isomorphic to $\left[\operatorname{cl}_{\|\cdot\|_{B D}}\left(B_{B D}\left(0, r_{2}\right)\right), \sigma\left(\mathbf{Y}^{1}(\bar{\Omega})\right.\right.$, $\left.\left.C_{\text {div }}\left(\bar{\Omega}, \mathbf{E}_{s}^{n}\right)\right)\right]$ (cf. [6, Proposition 4.25]).

Proposition 10. Every closed ball $\mathrm{cl}_{\|\cdot\|_{\mathbb{M}_{b}}}\left(B\left(0, r_{2}\right)\right)\left(\right.$ in $\left.\mathbf{Y}^{1}(\bar{\Omega})\right)$ is compact in the topology $\sigma\left(\mathbf{Y}^{1}(\bar{\Omega}), W^{n}(\Omega, \operatorname{div})\right)$. For $n=1, L^{n /(n-1)}\left(\Omega, \mathbb{R}^{n}\right)$ is replaced by $L^{\infty}\left(\Omega, \mathbb{R}^{1}\right)$ in the proof below.

Proof. Step 1. Let $\left\{\varepsilon\left(\mathbf{u}_{\delta}\right)_{\mid \bar{\Omega}}\right\}_{\delta \in D} \subset \mathbf{Y}^{1}(\bar{\Omega})$ be a bounded net in the norm $\|\cdot\|_{\mathbb{M}_{b}(\bar{\Omega})}$. Then $\left\{\mathbf{u}_{\delta \mid \Omega}\right\}_{\delta \in D} \subset B D(\Omega)$ is a $\|\cdot\|_{B D}$-bounded net. There exists a continuous injection of $B D(\Omega)$ into $L^{n /(n-1)}\left(\Omega, \mathbb{R}^{n}\right)$ (see $[23$, Chapter 2, Theorem 2.2]). Hence $\left\{\mathbf{u}_{\delta \mid \Omega}\right\}_{\delta \in D}$ is a bounded net in $L^{n /(n-1)}$. Therefore, there exists a finer net $\left\{\mathbf{u}_{\delta_{\alpha}}\right\}_{\alpha \in A} \subset\left\{\mathbf{u}_{\delta}\right\}_{\delta \in D}$ and a function $\mathbf{u}_{1} \in L^{n /(n-1)}\left(\Omega, \mathbb{R}^{n}\right)$ such that

$$
\left\langle\varepsilon\left(\mathbf{u}_{\delta_{\alpha}}\right), \boldsymbol{\sigma}\right\rangle_{\mathbf{Y}^{1} \times W^{n}(\Omega, \operatorname{div})}=-\int_{\Omega}(\operatorname{div} \boldsymbol{\sigma}) \cdot \mathbf{u}_{\delta_{\alpha}} d x \rightarrow-\int_{\Omega}(\operatorname{div} \boldsymbol{\sigma}) \cdot \mathbf{u}_{1} d x
$$

for every $\boldsymbol{\sigma} \in W^{n}\left(\Omega\right.$, div), since $\operatorname{div} \boldsymbol{\sigma} \in L^{n}\left(\Omega, \mathbb{R}^{n}\right)$. Moreover, there exists a finer net $\left\{\mathbf{u}_{\delta_{\alpha_{\beta}}}\right\}$ and a measure $\boldsymbol{\mu}_{1} \in \mathbb{M}_{b}\left(\Omega, \mathbf{E}_{s}^{n}\right)$ such that $\int_{\Omega} \boldsymbol{\varphi}: \boldsymbol{\varepsilon}\left(\mathbf{u}_{\delta_{\alpha_{\beta}}}\right) \rightarrow$ $\int_{\Omega} \boldsymbol{\varphi}: \boldsymbol{\mu}_{1}$ for every $\boldsymbol{\varphi} \in C_{0}^{1}\left(\Omega, \mathbf{E}_{s}^{n}\right)$. The symmetric distributional derivative $\varepsilon\left(\mathbf{u}_{1}\right)$ of $\mathbf{u}_{1}$ is equal to $\boldsymbol{\mu}_{1}$, since $C_{0}^{1}\left(\Omega_{1}, \mathbf{E}_{s}^{n}\right) \subset W^{n}\left(\Omega_{1}\right.$, div $)$. Thus $\mathbf{u}_{1} \in$ $B D(\Omega)$ and $\varepsilon\left(\mathbf{u}_{\delta_{\alpha_{\beta}}}\right)_{\mid \bar{\Omega}}$ converges to $\varepsilon\left(\widetilde{\mathbf{u}}_{1}\right)_{\mid \bar{\Omega}}$ in $\sigma\left(\mathbf{Y}^{1}(\bar{\Omega}), W^{n}(\Omega\right.$, div $\left.)\right)$, where $\widetilde{\mathbf{u}}_{1} \in B D\left(\Omega_{1}\right), \widetilde{\mathbf{u}}_{1 \mid \Omega}=\mathbf{u}_{1}$ in $\Omega$ and $\widetilde{\mathbf{u}}_{1 \mid \Omega_{1}-\bar{\Omega}}=\mathbf{0}$ in $\Omega_{1}-\bar{\Omega}$. 
Step 2. The net $\left\{\boldsymbol{\varepsilon}\left(\mathbf{u}_{\delta}\right)_{\mid \bar{\Omega}}\right\}_{\delta \in D} \subset \mathbf{Y}^{1}(\bar{\Omega})$ is contained in a closed, bounded ball $\mathrm{cl}_{\|\cdot\|_{\mathbb{M}_{b}}}\left(B\left(0, r_{2}\right)\right)$. Then for every $\delta \in D$,

$$
\begin{aligned}
\left\|\varepsilon\left(\mathbf{u}_{\delta}\right)_{\mid \bar{\Omega}}\right\|_{\mathbb{M}_{b}}=\sup \left\{\left\langle\varepsilon\left(\mathbf{u}_{\delta}\right), \boldsymbol{\sigma}\right\rangle_{\mathbf{Y}^{1} \times W^{n}\left(\Omega_{1}, \operatorname{div}\right)} \mid\right. & \\
\boldsymbol{\sigma} & \left.\in C_{0}^{1}\left(\Omega_{1}, \mathbf{E}_{s}^{n}\right),\|\boldsymbol{\sigma}(x)\|_{\mathbf{E}_{s}^{n}} \leq 1, \forall x \in \Omega_{1}\right\} \leq r_{2} .
\end{aligned}
$$

By (6.3) we get $\left\|\varepsilon\left(\widetilde{\mathbf{u}}_{1}\right)_{\mid \bar{\Omega}}\right\|_{\mathbb{M}_{b}} \leq r_{2}$. Then $\varepsilon\left(\widetilde{\mathbf{u}}_{1}\right)_{\mid \bar{\Omega}} \in \operatorname{cl}_{\|\cdot\|_{\mathbb{M}_{b}}}\left(B_{\mathbf{Y}^{1}(\bar{\Omega})}\left(0, r_{2}\right)\right)$.

Theorem 11. The topologies $\sigma\left(\mathbf{Y}^{1}(\bar{\Omega}), C_{\operatorname{div}}\left(\bar{\Omega}, \mathbf{E}_{s}^{n}\right)\right)$ and $\sigma\left(\mathbf{Y}^{1}(\bar{\Omega})\right.$, $W^{n}(\Omega$, div) $)$ are equivalent in every closed ball $\mathrm{cl}_{\|\cdot\|_{\mathbb{M}_{b}}}\left(B_{\mathbf{Y}^{1}(\bar{\Omega})}\left(0, r_{2}\right)\right)$.

Proof. The Hausdorff topology $\sigma\left(\mathbf{Y}^{1}(\bar{\Omega}), C_{\text {div }}\left(\bar{\Omega}, \mathbf{E}_{s}^{n}\right)\right)$ is weaker than $\sigma\left(\mathbf{Y}^{1}(\bar{\Omega}), W^{n}(\Omega, \operatorname{div})\right)$. Moreover, among all Hausdorff topologies, compact topologies are minimal (see [13, Corollary 3.1.14] and Proposition 10).

Assumption 5. There exists $\boldsymbol{\sigma}_{L} \in C_{\operatorname{div}}\left(\bar{\Omega}, \mathbf{E}_{s}^{n}\right)$ such that $\left\lceil P_{\lambda, r}^{*}\right\rceil\left(\boldsymbol{\sigma}_{L}\right)=$ $\sup \left(P_{\lambda, r}^{*}\right)<\infty, \boldsymbol{\beta}_{B}\left(\boldsymbol{\sigma}_{L}\right)=\lambda \mathbf{g}$ on $\Gamma_{1}$ and $\boldsymbol{\sigma}_{L}(x) \in \mathcal{K}(x)$. Moreover, there exists $\delta_{0}>0$ such that $\operatorname{dist}\left(\boldsymbol{\sigma}_{L}(x), \operatorname{Fr} \mathcal{K}(x)\right)=\inf \left\{\left\|\boldsymbol{\sigma}_{L}(x)-\mathbf{z}\right\|_{\mathbf{E}_{s}^{n}} \mid \mathbf{z} \in\right.$ $\operatorname{Fr} \mathcal{K}(x)\}>\delta_{0}$ for every $x \in \Omega$.

By Assumption 5 the boundary force $\mathbf{g} \in L^{\infty}\left(\Gamma_{1}, \mathbb{R}^{n}\right)$ is a regular function.

Define $T_{r}: \mathbf{Y}^{1}(\bar{\Omega}) \rightarrow \mathbb{R} \cup\{+\infty\}$ by

$$
\begin{aligned}
T_{r}\left(\varepsilon(\mathbf{u})_{\mid \bar{\Omega}}\right) \equiv & \int_{\operatorname{Fr} \Omega} \boldsymbol{\beta}_{B}\left(\boldsymbol{\sigma}_{L}\right) \cdot \gamma_{B}^{I}(\mathbf{u}) d s-\int_{\Omega} \boldsymbol{\sigma}_{L}: \boldsymbol{\varepsilon}(\mathbf{u})_{\mid \Omega} \\
& -\int_{\Gamma_{1}} \boldsymbol{\beta}_{B}\left(\boldsymbol{\sigma}_{L}\right) \boldsymbol{\gamma}_{B}^{I}(\mathbf{u}) d s+\int_{\Gamma_{1}} r\left\|\boldsymbol{\gamma}_{B}^{I}(\mathbf{u})\right\|_{\mathbb{R}^{n}}^{E} d s \\
& +\int_{\Gamma_{0}} I_{\left\{\gamma_{B}^{I}(\mathbf{u}) \otimes_{s} \boldsymbol{\nu}=0\right\}}\left(-\boldsymbol{\gamma}_{B}^{I}(\mathbf{u}) \otimes_{s} \boldsymbol{\nu}\right) d s+\int_{\Omega} j(x, \boldsymbol{\varepsilon}(\mathbf{u})) d x
\end{aligned}
$$

if $\mathbf{u}_{\mid \Omega} \in L D(\Omega)$ and $\mathbf{u}_{\mid \Omega_{1}-\bar{\Omega}}=\mathbf{0}$, and $T_{r}\left(\varepsilon(\mathbf{u})_{\mid \bar{\Omega}}\right) \equiv+\infty$ otherwise. By (2.11) we have $T_{r}\left(\varepsilon(\mathbf{u})_{\mid \bar{\Omega}}\right)=\lambda F_{r}\left(\mathbf{u}_{\mid \Omega}\right)+G_{j}\left(\varepsilon\left(\mathbf{u}_{\mid \Omega}\right)\right)$ if $\mathbf{u}_{\mid \Omega} \in L D(\Omega)$ and $\mathbf{u}_{\mid \Omega_{1}-\bar{\Omega}}=\mathbf{0}$.

Because of the duality between $\mathbf{Y}^{1}(\bar{\Omega})$ and $W^{n}(\Omega$, div), we define a functional $T_{\lambda}^{*}$ on the linear space $C_{\operatorname{div}}\left(\bar{\Omega}, \mathbf{E}_{s}^{n}\right)+\left\{\boldsymbol{\sigma} \in W^{n}(\Omega, \operatorname{div}) \mid \operatorname{div} \boldsymbol{\sigma}=\mathbf{0}\right\}$ by

$$
\begin{array}{r}
T_{r}^{*}(\boldsymbol{\sigma})=\sup \left\{\left\langle\varepsilon(\mathbf{u})_{\mid \bar{\Omega}}, \boldsymbol{\sigma}\right\rangle_{\mathbf{Y}^{1} \times W^{n}(\Omega, \text { div })}-T_{r}\left(\varepsilon(\mathbf{u})_{\mid \bar{\Omega}}\right) \mid \mathbf{u} \in B D\left(\Omega_{1}\right),\right. \\
\left.\mathbf{u}_{\mid \Omega} \in L D(\Omega) \text { and } \mathbf{u}_{\mid \Omega_{1}-\bar{\Omega}}=\mathbf{0}\right\} .
\end{array}
$$

The bidual functional $T_{r}^{* *}: \mathbf{Y}^{1}(\bar{\Omega}) \rightarrow \mathbb{R} \cup\{+\infty\}$ is defined by

$$
\begin{aligned}
T_{r}^{* *}\left(\varepsilon(\mathbf{u})_{\mid \bar{\Omega}}\right)= & \sup \left\{\left\langle\varepsilon(\mathbf{u})_{\mid \bar{\Omega}}, \boldsymbol{\sigma}\right\rangle_{\mathbf{Y}^{1} \times W^{n}(\Omega, \operatorname{div})}-T_{r}^{*}(\boldsymbol{\sigma}) \mid\right. \\
& \left.\boldsymbol{\sigma} \in C_{\operatorname{div}}\left(\bar{\Omega}, \mathbf{E}_{s}^{n}\right)+\left\{\boldsymbol{\sigma}_{s} \in W^{n}(\Omega, \operatorname{div}) \mid \operatorname{div} \boldsymbol{\sigma}_{s}=\mathbf{0}\right\}\right\}
\end{aligned}
$$

for $\mathbf{u} \in B D\left(\Omega_{1}\right)$ with $\mathbf{u}_{\mid \Omega_{1}-\bar{\Omega}}=\mathbf{0}$. 
Because of (3.12), the space $\mathbf{Y}^{1}(\bar{\Omega})_{\mid \operatorname{Fr} \Omega}$ is isomorphic to $\left\{-\gamma_{B}(\mathbf{u}) \otimes_{s} \boldsymbol{\nu} \in\right.$ $\left.L^{1}\left(\operatorname{Fr} \Omega, \mathbf{E}_{s}^{n}\right) \mid \mathbf{u} \in B D(\Omega)\right\}$. The extension $\tilde{\mathbf{Y}}^{1}(\bar{\Omega})$ of $\mathbf{Y}^{1}(\bar{\Omega})$ is given by

$$
\begin{array}{r}
\widetilde{\mathbf{Y}}^{1}(\bar{\Omega}) \equiv\left\{\left(\mathbf{z},-\boldsymbol{\gamma}_{B}^{I}(\mathbf{u}) \otimes_{s} \boldsymbol{\nu}\right)\right. \\
\in \operatorname{span}\left(\varepsilon(B D(\Omega)), L^{1}\left(\Omega, \mathbf{E}_{s}^{n}\right)\right) \times \mathbf{Y}^{1}(\bar{\Omega})_{\mid \operatorname{Fr} \Omega} \mid \\
\exists \mathbf{w} \in L^{1}\left(\Omega, \mathbf{E}_{s}^{n}\right), \quad \exists \widetilde{\mathbf{u}} \in B D(\Omega) \text { such that } \mathbf{z}=\mathbf{w} d x+\varepsilon(\widetilde{\mathbf{u}}) \\
\text { and } \left.\gamma_{B}^{I}(\mathbf{u}) \otimes_{s} \boldsymbol{\nu}=\gamma_{B}(\widetilde{\mathbf{u}}) \otimes_{s} \boldsymbol{\nu}\right\} .
\end{array}
$$

The bilinear pairing between $\widetilde{\mathbf{Y}}^{1}(\bar{\Omega})$ and $W^{n}(\Omega, \operatorname{div})$ is given by

$$
\left\langle\left(\mathbf{z},-\boldsymbol{\gamma}_{B}^{I}(\mathbf{u}) \otimes_{s} \boldsymbol{\nu}\right), \boldsymbol{\sigma}\right\rangle_{1} \equiv \int_{\Omega} \boldsymbol{\sigma}: \mathbf{z}-\int_{\operatorname{Fr} \Omega} \boldsymbol{\beta}_{B}(\boldsymbol{\sigma}) \cdot \boldsymbol{\gamma}_{B}^{I}(\mathbf{u}) d s
$$

for every $\boldsymbol{\sigma} \in W^{n}(\Omega, \operatorname{div})$ and every $\left(\mathbf{z},-\gamma_{B}^{I}(\mathbf{u}) \otimes_{s} \boldsymbol{\nu}\right) \in \widetilde{\mathbf{Y}}^{1}(\bar{\Omega})$. A net $\left\{\mathbf{M}_{t}\right\}_{t \in T} \subset \tilde{\mathbf{Y}}^{1}(\bar{\Omega})$ is convergent to $\mathbf{M}_{0}$ in $\sigma\left(\tilde{\mathbf{Y}}^{1}(\bar{\Omega}), W^{n}(\Omega, \operatorname{div})\right)$ if and only if $\left\langle\mathbf{M}_{t}, \boldsymbol{\sigma}\right\rangle_{1} \rightarrow\left\langle\mathbf{M}_{0}, \boldsymbol{\sigma}\right\rangle_{1}$ for all $\boldsymbol{\sigma} \in W^{n}\left(\Omega_{1}\right.$, div). The extension of $T_{r}$ onto the space $\widetilde{\mathbf{Y}}^{1}(\bar{\Omega})$ (denoted by $\widetilde{T}_{r}$ ) is given by

$$
\begin{aligned}
& \widetilde{T}_{r}\left(\mathbf{z},-\boldsymbol{\gamma}_{B}(\mathbf{u}) \otimes_{s} \boldsymbol{\nu}\right) \\
\equiv- & -\left\langle\left(\mathbf{z},-\boldsymbol{\gamma}_{B}(\mathbf{u}) \otimes_{s} \boldsymbol{\nu}\right), \boldsymbol{\sigma}_{L}\right\rangle_{1}-\int_{\Gamma_{1}} \boldsymbol{\beta}_{B}\left(\boldsymbol{\sigma}_{L}\right) \boldsymbol{\gamma}_{B}(\mathbf{u}) d s+\int_{\Gamma_{1}} r\left\|\boldsymbol{\gamma}_{B}^{I}(\mathbf{u})\right\|_{\mathbb{R}^{n}}^{E} d s \\
+ & \int_{\Gamma_{0}} I_{\left\{\gamma_{B}(\mathbf{u}) \otimes_{s} \boldsymbol{\nu}=\mathbf{0}\right\}}\left(-\boldsymbol{\gamma}_{B}(\mathbf{u}) \otimes_{s} \boldsymbol{\nu}\right) d s+\int_{\Omega} j(x, \mathbf{z}) d x
\end{aligned}
$$

if $\mathbf{z}=\mathbf{w} d x+\varepsilon(\mathbf{u})$ with $\mathbf{w} \in L^{1}\left(\Omega, \mathbf{E}_{s}^{n}\right), \mathbf{u} \in L D(\Omega)$, and $\widetilde{T}_{r}\left(\mathbf{z},-\gamma_{B}(\mathbf{u}) \otimes_{s} \boldsymbol{\nu}\right)$ $\equiv+\infty$ otherwise.

By the duality between $\tilde{\mathbf{Y}}^{1}(\bar{\Omega})$ and $W^{n}(\Omega$, div $)$ we define a functional $\widetilde{T}_{r}^{*}$ on the linear space $C_{\operatorname{div}}\left(\bar{\Omega}, \mathbf{E}_{s}^{n}\right)+\left\{\boldsymbol{\sigma} \in W^{n}(\Omega, \operatorname{div}) \mid \operatorname{div} \boldsymbol{\sigma}=\mathbf{0}\right\}$ by

$$
\begin{aligned}
\widetilde{T}_{r}^{*}(\boldsymbol{\sigma})=\sup \left\{\left\langle\left(\mathbf{z},-\boldsymbol{\gamma}_{B}(\mathbf{u}) \otimes_{s} \boldsymbol{\nu}\right), \boldsymbol{\sigma}\right\rangle_{1}-\widetilde{T}_{r}\left(\mathbf{z},-\boldsymbol{\gamma}_{B}(\mathbf{u}) \otimes_{s} \boldsymbol{\nu}\right) \mid\right. \\
\left.\mathbf{z} \in L^{1}\left(\Omega, \mathbf{E}_{s}^{n}\right), \mathbf{u} \in L D(\Omega)\right\} .
\end{aligned}
$$

The bidual functional $\widetilde{T}_{r}^{* *}: \widetilde{\mathbf{Y}}^{1}(\bar{\Omega}) \rightarrow \mathbb{R} \cup\{+\infty\}$ is defined by

$$
\begin{array}{r}
\widetilde{T}_{r}^{* *}\left(\mathbf{z},-\boldsymbol{\gamma}_{B}(\mathbf{u}) \otimes_{s} \boldsymbol{\nu}\right)=\sup \left\{\left\langle\left(\mathbf{z},-\boldsymbol{\gamma}_{B}(\mathbf{u}) \otimes_{s} \boldsymbol{\nu}\right), \boldsymbol{\sigma}\right\rangle_{1}-T_{r}^{*}(\boldsymbol{\sigma}) \mid\right. \\
\left.\boldsymbol{\sigma} \in C_{\operatorname{div}}\left(\bar{\Omega}, \mathbf{E}_{s}^{n}\right)+\left\{\boldsymbol{\sigma} \in W^{n}(\Omega, \operatorname{div}) \mid \operatorname{div} \boldsymbol{\sigma}=\mathbf{0}\right\}\right\}
\end{array}
$$

for $\left(\mathbf{z},-\gamma_{B}(\mathbf{u}) \otimes_{s} \boldsymbol{\nu}\right) \in \tilde{\mathbf{Y}}^{1}(\bar{\Omega})$.

Proposition 12. The explicit form of $\widetilde{T}_{r}^{*}$ is

$$
\widetilde{T}_{r}^{*}(\boldsymbol{\sigma})=\int_{\Omega} j^{*}\left(x, \boldsymbol{\sigma}+\boldsymbol{\sigma}_{L}\right) d x+\int_{\Gamma_{1}} I_{B^{E}(0, r)}\left(-\boldsymbol{\beta}_{B}(\boldsymbol{\sigma})\right) d s
$$

for every $\boldsymbol{\sigma} \in C_{\operatorname{div}}\left(\bar{\Omega}, \mathbf{E}_{s}^{n}\right)+\left\{\boldsymbol{\sigma} \in W^{n}(\Omega, \operatorname{div}) \mid \operatorname{div} \boldsymbol{\sigma}=\mathbf{0}\right\}$. Here $B^{E}(0, r)$ is the closed ball in $\mathbb{R}^{n}$, endowed with the Euclidean norm $\|\cdot\|_{\mathbb{R}^{n}}^{E}$. 
If we extend $\widetilde{T}_{r}^{*}$ onto the space $W^{n}(\Omega, \operatorname{div})$ by $(6.15)$, then $\widetilde{T}_{r}^{*}$ is given by (6.17) for every $\boldsymbol{\sigma} \in W^{n}(\Omega, \operatorname{div})$.

Proof. By Theorem 3A of [21] and formulae (6.14), (6.15), we have

$$
\begin{aligned}
& \widetilde{T}_{r}^{*}(\boldsymbol{\sigma})=\sup \left\{\int_{\Omega}\left(\boldsymbol{\sigma}+\boldsymbol{\sigma}_{L}\right): \mathbf{z} d x-\int_{\operatorname{Fr} \Omega} \boldsymbol{\beta}_{B}\left(\boldsymbol{\sigma}+\boldsymbol{\sigma}_{L}\right) \cdot \boldsymbol{\gamma}_{B}(\mathbf{u}) d s\right. \\
& +\int_{\Gamma_{1}} \boldsymbol{\beta}_{B}\left(\boldsymbol{\sigma}_{L}\right) \cdot \boldsymbol{\gamma}_{B}(\mathbf{u}) d s-\int_{\Gamma_{1}} r\left\|\boldsymbol{\gamma}_{B}^{I}(\mathbf{u})\right\|_{\mathbb{R}^{n}}^{E} d s \\
& -\int_{\Gamma_{0}} I_{\left\{\gamma_{B}(\mathbf{u}) \otimes_{s} \boldsymbol{\nu}=\mathbf{0}\right\}}\left(-\boldsymbol{\gamma}_{B}(\mathbf{u}) \otimes_{s} \boldsymbol{\nu}\right) d s-\int_{\Omega} j(x, \mathbf{z}) d x \mid \\
& \left.\mathbf{z}=\mathbf{w}+\boldsymbol{\varepsilon}(\mathbf{u}), \text { where } \mathbf{w} \in L^{1}\left(\Omega, \mathbf{E}_{s}^{n}\right) \text { and } \mathbf{u} \in L D(\Omega)\right\} \\
& =\sup \left\{\int_{\Omega}\left(\boldsymbol{\sigma}+\boldsymbol{\sigma}_{L}\right): \mathbf{w} d x-\int_{\Omega} j(x, \mathbf{w}) d x \mid \mathbf{w} \in L^{1}\left(\Omega, \mathbf{E}_{s}^{n}\right)\right\} \\
& +\sup \left\{-\int_{\operatorname{Fr} \Omega} \boldsymbol{\beta}_{B}\left(\boldsymbol{\sigma}+\boldsymbol{\sigma}_{L}\right) \cdot \boldsymbol{\gamma}_{B}(\mathbf{u}) d s+\int_{\Gamma_{1}} \boldsymbol{\beta}_{B}\left(\boldsymbol{\sigma}_{L}\right) \cdot \boldsymbol{\gamma}_{B}(\mathbf{u}) d s\right. \\
& \left.-\int_{\Gamma_{1}} r\left\|\boldsymbol{\gamma}_{B}^{I}(\mathbf{u})\right\|_{\mathbb{R}^{n}}^{E} d s \mid \boldsymbol{\gamma}_{B}(\mathbf{u}) \in L^{1}\left(\operatorname{Fr} \Omega, \mathbb{R}^{n}\right) \text { and } \boldsymbol{\gamma}_{B}(\mathbf{u})=\mathbf{0} \text { on } \Gamma_{0}\right\}
\end{aligned}
$$

for every $\boldsymbol{\sigma} \in W^{n}\left(\Omega\right.$, div), because $\gamma_{B}$ is a surjection on $L^{1}\left(\operatorname{Fr} \Omega, \mathbb{R}^{n}\right)$. Then we obtain (6.17).

Define $\widetilde{T}_{r}^{* \#}: \widetilde{\mathbf{Y}}^{1}(\bar{\Omega}) \rightarrow \mathbb{R} \cup\{+\infty\}$ by

$$
\begin{aligned}
& \widetilde{T}_{r}^{* \#}\left(\mathbf{z},-\gamma_{B}(\mathbf{u}) \otimes_{s} \boldsymbol{\nu}\right) \equiv \sup \left\{\left\langle\left(\mathbf{z},-\gamma_{B}(\mathbf{u}) \otimes_{s} \boldsymbol{\nu}\right),\right.\right.\boldsymbol{\sigma}\rangle_{1}-\widetilde{T}_{r}^{*}(\boldsymbol{\sigma}) \mid \\
&\left.\boldsymbol{\sigma} \in C_{\mathrm{div}}\left(\bar{\Omega}, \mathbf{E}_{s}^{n}\right)\right\}
\end{aligned}
$$

for every $\left(\mathbf{z},-\gamma_{B}(\mathbf{u}) \otimes_{s} \boldsymbol{\nu}\right) \in \tilde{\mathbf{Y}}^{1}(\bar{\Omega})$.

Proposition 13. The explicit form of $\widetilde{T}_{r}^{* \#}$ is

$$
\begin{aligned}
\widetilde{T}_{r}^{* \#}\left(\boldsymbol{\varepsilon}(\mathbf{u})_{\mid \bar{\Omega}}\right) & \\
= & -\lambda \int_{\Omega} \mathbf{f} \cdot \mathbf{u} d x-\int_{\Gamma_{1}} \boldsymbol{\beta}_{B}\left(\boldsymbol{\sigma}_{L}\right) \cdot \gamma_{B}^{I}(\mathbf{u}) d s \\
& +\int_{\Gamma_{1}} r\left\|\boldsymbol{\gamma}_{B}^{I}(\mathbf{u})\right\|_{\mathbb{R}^{n}}^{E} d s+\int_{\Gamma_{0}} j_{\infty}\left(x,-\gamma_{B}^{I}(\mathbf{u}) \otimes_{s} \boldsymbol{\nu}\right) d s \\
& +\int_{\Omega} j\left(x, \boldsymbol{\varepsilon}(\mathbf{u})_{a}\right) d x+\int_{\Omega} j_{\infty}\left(x, d \boldsymbol{\varepsilon}(\mathbf{u})_{s} / d\left|\boldsymbol{\varepsilon}(\mathbf{u})_{s}\right|\right) d\left|\boldsymbol{\varepsilon}(\mathbf{u})_{s}\right|
\end{aligned}
$$

for $\boldsymbol{\varepsilon}(\mathbf{u})_{\mid \bar{\Omega}} \in \mathbf{Y}^{1}(\bar{\Omega})$.

Proof. The field $\boldsymbol{\sigma}_{L}$ is a solution of $\left(P_{\lambda, r}^{*}\right)$, i.e. $\left(P_{\lambda, r}^{*}\right)\left(\boldsymbol{\sigma}_{L}\right)=\sup \left(P_{\lambda, r}^{*}\right)$ (cf. (6.1)). Then $\widetilde{T}_{r}^{*}(\mathbf{0})<\infty$. Moreover, the space $C_{\operatorname{div}}\left(\bar{\Omega}, \mathbf{E}_{s}^{n}\right)$ is $P C U$-stable, so 
by Theorem 1 of [8] we get

$$
\begin{aligned}
& \widetilde{T}_{r}^{* \#}\left(\varepsilon(\mathbf{u})_{\mid \bar{\Omega}}\right)=\sup \left\{\int_{\Omega}\left(\boldsymbol{\sigma}+\boldsymbol{\sigma}_{L}\right): \varepsilon(\mathbf{u})-\int_{\Omega} j^{*}\left(x, \boldsymbol{\sigma}+\boldsymbol{\sigma}_{L}\right) d x\right. \\
& -\int_{\Gamma_{0}} \boldsymbol{\beta}_{B}\left(\boldsymbol{\sigma}+\boldsymbol{\sigma}_{L}\right) \cdot \gamma_{B}^{I}(\mathbf{u}) d s-\int_{\Gamma_{1}} \boldsymbol{\beta}_{B}\left(\boldsymbol{\sigma}+\boldsymbol{\sigma}_{L}\right) \cdot \gamma_{B}^{I}(\mathbf{u}) d s \\
& -\int_{\Gamma_{1}} I_{B^{E}(0, r)}\left(-\boldsymbol{\beta}_{B}(\boldsymbol{\sigma})\right) d s \\
& \left.\boldsymbol{\sigma} \in C_{\operatorname{div}}\left(\bar{\Omega}, \mathbf{E}_{s}^{n}\right),\left(\boldsymbol{\sigma}+\lambda_{L} \boldsymbol{\sigma}_{L}\right)(x) \in \mathcal{K}(x) \text { for every } x \in \bar{\Omega}\right\} \\
& +\int_{\operatorname{Fr} \Omega} \boldsymbol{\beta}_{B}\left(\boldsymbol{\sigma}_{L}\right) \cdot \gamma_{B}^{I}(\mathbf{u}) d s-\int_{\Omega} \boldsymbol{\sigma}_{L}: \boldsymbol{\varepsilon}(\mathbf{u}) \\
& \sup \left\{\int_{\Omega}\left[\left(\boldsymbol{\sigma}+\boldsymbol{\sigma}_{L}\right): \boldsymbol{\varepsilon}(\mathbf{u})_{a}-j^{*}\left(x, \boldsymbol{\sigma}+\boldsymbol{\sigma}_{L}\right)\right] d x\right.
\end{aligned}
$$$$
+\int_{\Omega}\left[\left(\boldsymbol{\sigma}+\boldsymbol{\sigma}_{L}\right):\left(d \varepsilon(\mathbf{u})_{s} / d\left|\varepsilon(\mathbf{u})_{s}\right|\right)-j_{\infty}^{*}\left(x, \boldsymbol{\sigma}+\boldsymbol{\sigma}_{L}\right)\right] d\left|\varepsilon(\mathbf{u})_{s}\right|
$$$$
+\int_{\Gamma_{0}}\left[\left(\boldsymbol{\sigma}+\boldsymbol{\sigma}_{L}\right):\left(-\boldsymbol{\gamma}_{B}^{I}(\mathbf{u}) \otimes_{s} \boldsymbol{\nu}\right)-j_{\infty}^{*}\left(x, \boldsymbol{\sigma}+\boldsymbol{\sigma}_{L}\right)\right] d s
$$$$
+\int_{\Gamma_{1}}\left[\left(-\boldsymbol{\beta}_{B}(\boldsymbol{\sigma})\right) \cdot \gamma_{B}^{I}(\mathbf{u})-I_{B^{E}(0, r)}\left(-\boldsymbol{\beta}_{B}(\boldsymbol{\sigma})\right)\right] d s-\int_{\Gamma_{1}} \boldsymbol{\beta}_{B}\left(\boldsymbol{\sigma}_{L}\right) \cdot \boldsymbol{\gamma}_{B}^{I}(\mathbf{u}) d s
$$$$
\left.\boldsymbol{\sigma} \in C_{\operatorname{div}}\left(\bar{\Omega}, \mathbf{E}_{s}^{n}\right)\right\}-\lambda \int_{\Omega} \mathbf{f} \cdot \mathbf{u} d x
$$

for every $\mathbf{u} \in B D\left(\Omega_{1}\right)$ such that $\mathbf{u}_{\mid \Omega_{1}-\bar{\Omega}}=\mathbf{0}$, which is $(6.20)$. Here $\boldsymbol{\varepsilon}(\mathbf{u})=$ $\varepsilon(\mathbf{u})_{a}+\varepsilon(\mathbf{u})_{s}$ is the Lebesgue decomposition of $\varepsilon(\mathbf{u})$ into absolutely continuous and singular parts with respect to $d x$.

Definition 4. We say that $T_{r}$ is coercive if

$$
T_{r}\left(\mathbf{M}_{m}\right) \rightarrow+\infty \quad \text { if }\left\|\mathbf{M}_{m}\right\|_{\mathbb{M}_{b}\left(\bar{\Omega}, \mathbf{E}_{s}^{n}\right)} \rightarrow+\infty
$$

for every sequence $\left\{\mathbf{M}_{m}\right\}_{m \in \mathbb{N}} \subset \mathbf{Y}^{1}(\bar{\Omega})$.

Assumption 6. Let $T_{r}$ be a coercive function. Moreover, let $\widetilde{T}_{r}^{* \#}$ be the largest minorant that is less than $T_{r}$ and l.s.c. in the topology $\sigma\left(\mathbf{Y}^{1}(\bar{\Omega})\right.$, $\left.C_{\text {div }}\left(\bar{\Omega}, \mathbf{E}_{s}^{n}\right)\right)$, or in other words, let $\widetilde{T}_{r}^{* \#}$ be the l.s.c. regularization of $T_{r}$ in $\sigma\left(\mathbf{Y}^{1}(\bar{\Omega}), C_{\operatorname{div}}\left(\bar{\Omega}, \mathbf{E}_{s}^{n}\right)\right)$.

REMARK 4. Since $T_{r}$ is a coercive function on $\mathbf{Y}^{1}(\bar{\Omega})$, it suffices to consider equivalent topologies in every closed ball $\mathrm{cl}_{\|\cdot\|_{\mathbb{M}_{b}}}\left(B_{\mathbf{Y}^{1}(\bar{\Omega})}\left(0, r_{2}\right)\right)$. Because of Theorem 11, the topologies $\sigma\left(\mathbf{Y}^{1}(\bar{\Omega}), C_{\operatorname{div}}\left(\bar{\Omega}, \mathbf{E}_{s}^{n}\right)\right)$ and $\sigma\left(\mathbf{Y}^{1}(\bar{\Omega})\right.$, $\left.W^{n}(\Omega, \operatorname{div})\right)$ are equivalent in every closed ball in $\mathbf{Y}^{1}(\bar{\Omega})$. 
We say that a net $\left\{\mathbf{M}_{\delta}\right\}_{\delta \in D} \subset \mathbf{Y}^{1}(\bar{\Omega})$ is convergent to $\mathbf{M}_{0}$ in the topology $\sigma\left(\mathbf{Y}^{1}(\bar{\Omega}), C_{\operatorname{div}}\left(\bar{\Omega}, \mathbf{E}_{s}^{n}\right)+\left\{\boldsymbol{\sigma} \in W^{n}(\Omega, \operatorname{div}) \mid \operatorname{div} \boldsymbol{\sigma}=\mathbf{0}\right\}\right)$ if $\left\langle\mathbf{M}_{\delta}-\mathbf{M}_{0}\right.$, $\boldsymbol{\sigma}\rangle_{\mathbf{Y}^{1} \times W^{n}(\Omega, \text { div })} \rightarrow 0$ for all $\boldsymbol{\sigma} \in C_{\text {div }}\left(\bar{\Omega}, \mathbf{E}_{s}^{n}\right)+\left\{\boldsymbol{\sigma} \in W^{n}(\Omega, \operatorname{div}) \mid \operatorname{div} \boldsymbol{\sigma}=\mathbf{0}\right\}$. The topologies $\sigma\left(\mathbf{Y}^{1}(\bar{\Omega}), C_{\operatorname{div}}\left(\bar{\Omega}, \mathbf{E}_{s}^{n}\right)+\left\{\boldsymbol{\sigma} \in W^{n}(\Omega, \operatorname{div}) \mid \operatorname{div} \boldsymbol{\sigma}=\mathbf{0}\right\}\right)$ and $\sigma\left(\mathbf{Y}^{1}(\bar{\Omega}), W^{n}(\Omega, \operatorname{div})\right)$ are equivalent in every closed ball in $\mathbf{Y}^{1}(\bar{\Omega})$, because $\sigma\left(\mathbf{Y}^{1}(\bar{\Omega}), C_{\operatorname{div}}\left(\bar{\Omega}, \mathbf{E}_{s}^{n}\right)+\left\{\boldsymbol{\sigma} \in W^{n}(\Omega, \operatorname{div}) \mid \operatorname{div} \boldsymbol{\sigma}=\mathbf{0}\right\}\right)$ is weaker than $\sigma\left(\mathbf{Y}^{1}(\bar{\Omega}), W^{n}(\Omega, \operatorname{div})\right)$ and stronger than $\sigma\left(\mathbf{Y}^{1}(\bar{\Omega}), C_{\operatorname{div}}\left(\bar{\Omega}, \mathbf{E}_{s}^{n}\right)\right)$.

By Proposition 2 and $[6$, Proposition 4.25$]$ the spaces $\left[\operatorname{cl}_{\|\cdot\|_{B D}}\left(B_{B D}\left(0, r_{2}\right)\right)\right.$, $\left.\|\cdot\|_{L^{p}\left(\Omega, \mathbb{R}^{n}\right)}\right]$ and $\left[\mathrm{cl}_{\|\cdot\|_{B D}}\left(B_{B D}\left(0, r_{2}\right)\right), \sigma\left(\mathbf{Y}^{1}(\bar{\Omega}), W^{n}(\Omega, \operatorname{div})\right)\right]$ are homeomorphic for $1 \leq p<q=n /(n-1)$ (if $n=1$ then $q=\infty$ ), where the isomorphism between $B D(\Omega)$ and $\mathbf{Y}^{1}(\bar{\Omega})$ is given by (6.7). Indeed, $\left[\mathrm{cl}_{\|\cdot\|_{B D}}\left(B_{B D}\left(0, r_{2}\right)\right),\|\cdot\|_{L^{p}\left(\Omega, \mathbb{R}^{n}\right)}\right]$ is a Hausdorff topological space and the topology given by the norm $\|\cdot\|_{L^{p}\left(\Omega, \mathbb{R}^{n}\right)}$ is weaker than the topology (2.7)(2.8) (see Definition 1 and [13, Corollary 3.1.14]).

Let $T_{0}$ denote $T_{r}$ for $r=0$ (cf. (6.9)). The problem of l.s.c. regularization of $T_{0}$, for the homogeneous case, is solved in [9] and [4]; the regularization is found in the topology of $L^{1}\left(\Omega, \mathbb{R}^{n}\right)$. The non-homogeneous case is studied in [7] for functionals defined in the space $B V$ of vector fields with bounded variation.

Lemma 14. For every $\boldsymbol{\sigma} \in C_{\operatorname{div}}\left(\bar{\Omega}, \mathbf{E}_{s}^{n}\right)+\left\{\boldsymbol{\sigma} \in W^{n}(\Omega, \operatorname{div}) \mid \operatorname{div} \boldsymbol{\sigma}=\mathbf{0}\right\}$ we have $\widetilde{T}_{r}^{*}(\boldsymbol{\sigma}) \geq T_{r}^{*}(\boldsymbol{\sigma})$. Moreover, $\widetilde{T}_{r}^{* *}(\mathbf{M}) \leq T_{r}^{* *}(\mathbf{M})$ for every $\mathbf{M} \in$ $\mathbf{Y}^{1}(\bar{\Omega})$.

Proof. Indeed, in the definition of $\widetilde{T}_{r}^{*}$ we take the supremum over a larger domain. The second inequality follows directly from the first.

Proposition 15. Under Assumption 6, we have $\widetilde{T}_{r}^{* \#}(\mathbf{M})=\widetilde{T}_{r}^{* *}(\mathbf{M})=$ $T_{r}^{* *}(\mathbf{M})$ for every $\mathbf{M} \in \mathbf{Y}^{1}(\bar{\Omega})$.

Proof. By Lemma 14, $\widetilde{T}_{r}^{* *} \leq T_{r}^{* *}$. Moreover, in the definition of $\widetilde{T}_{r}^{* *}$ we take the supremum over a larger domain (see (6.19) and (6.16)), so $\widetilde{T}_{r}^{* \#}(\mathbf{M}) \leq \widetilde{T}_{r}^{* *}(\mathbf{M})$ for every $\mathbf{M} \in \mathbf{Y}^{1}(\bar{\Omega})$. Therefore $\widetilde{T}_{r}^{* \#} \leq \widetilde{T}_{r}^{* *} \leq T_{r}^{* *}$. By (6.10), (6.11) and Remark 4, the functional $T_{r}^{* *}$ is the l.s.c. regularization of $T_{r}$ in the topology $\sigma\left(\mathbf{Y}^{1}(\bar{\Omega}), C_{\operatorname{div}}\left(\bar{\Omega}, \mathbf{E}_{s}^{n}\right)\right)$. Because of Assumption $6, \widetilde{T}_{r}^{* \#}$ is the l.s.c. regularization of $T_{r}$. Therefore $\widetilde{T}_{r}^{* \#}=T_{r}^{* *}$.

Lemma 16. Let Assumption 6 hold. For every $\mathbf{u} \in B D\left(\Omega_{1}\right)$ such that $\mathbf{u}_{\mid \Omega} \in L D(\Omega), \mathbf{u}_{\mid \Omega_{1}-\bar{\Omega}}=\mathbf{0}$ and $\boldsymbol{\gamma}_{B}^{I}(\mathbf{u})_{\mid \Gamma_{0}}=0$, we have

$$
\widetilde{T}_{r}^{* *}\left(\varepsilon(\mathbf{u})_{\mid \bar{\Omega}}\right)=T_{r}^{* *}\left(\varepsilon(\mathbf{u})_{\mid \bar{\Omega}}\right)=T_{r}\left(\varepsilon(\mathbf{u})_{\mid \bar{\Omega}}\right) .
$$

Proof. By Lemma 14, we get $\widetilde{T}_{r}^{* *} \leq T_{r}^{* *}$. Thus $\widetilde{T}_{r}^{* *}(\mathbf{M}) \leq T_{r}^{* *}(\mathbf{M}) \leq$ $T_{r}(\mathbf{M})$ for every $\mathbf{M} \in \mathbf{Y}^{1}(\bar{\Omega})$. Therefore, by (6.20) and Proposition 15, we get (6.23). 
Lemma 17. For every $\boldsymbol{\sigma} \in C_{\mathrm{div}}\left(\bar{\Omega}, \mathbf{E}_{s}^{n}\right)+\left\{\boldsymbol{\sigma} \in W^{n}(\Omega, \operatorname{div}) \mid \operatorname{div} \boldsymbol{\sigma}=\mathbf{0}\right\}$ and every $\boldsymbol{\sigma}_{s} \in W^{n}\left(\Omega\right.$, div) such that $\operatorname{div} \boldsymbol{\sigma}_{s}=\mathbf{0}$, we have

$$
T_{r}^{*}(\boldsymbol{\sigma})=T_{r}^{*}\left(\boldsymbol{\sigma}+\boldsymbol{\sigma}_{s}\right) .
$$

Proof. By (6.3), (6.10) and by Green's formula (2.11) we get

$$
\begin{aligned}
& T_{r}^{*}(\boldsymbol{\sigma})=\sup \left\{-\int_{\Omega}(\operatorname{div} \boldsymbol{\sigma}) \cdot \mathbf{u} d x-T_{r}\left(\varepsilon(\mathbf{u})_{\mid \bar{\Omega}}\right) \mid\right. \\
&\left.\mathbf{u}_{\mid \Omega} \in L D(\Omega) \text { and } \mathbf{u}_{\mid \Omega_{1}-\bar{\Omega}}=\mathbf{0}\right\} \\
&=\sup \left\{-\int_{\Omega}\left[\operatorname{div}\left(\boldsymbol{\sigma}+\boldsymbol{\sigma}_{s}\right)\right] \cdot \mathbf{u} d x-T_{r}\left(\varepsilon(\mathbf{u})_{\mid \bar{\Omega}}\right) \mid\right. \\
&\left.\mathbf{u}_{\mid \Omega} \in L D(\Omega) \text { and } \mathbf{u}_{\mid \Omega_{1}-\bar{\Omega}}=\mathbf{0}\right\} \\
&=T_{r}^{*}\left(\boldsymbol{\sigma}+\boldsymbol{\sigma}_{s}\right) .
\end{aligned}
$$

We say that a net $\left\{\boldsymbol{\sigma}_{k}\right\}_{k \in K} \subset W^{n}\left(\Omega\right.$,div) converges to $\widehat{\boldsymbol{\sigma}} \in W^{n}(\Omega, \operatorname{div})$ in the topology

$$
\sigma\left(W^{n}(\Omega, \operatorname{div}), L^{\infty}\left(\Omega, \mathbf{E}_{s}^{n}\right) \times L^{\infty}\left(\Gamma_{1}, \mathbb{R}^{n}\right)\right)
$$

if

$$
\int_{\Omega}\left(\boldsymbol{\sigma}_{k}-\widehat{\boldsymbol{\sigma}}\right): \mathbf{w} d x+\int_{\Gamma_{1}} \boldsymbol{\beta}_{B}\left(\boldsymbol{\sigma}_{k}-\widehat{\boldsymbol{\sigma}}\right) \cdot \mathbf{h} d s \rightarrow 0
$$

for every $\mathbf{w} \in L^{\infty}\left(\Omega, \mathbf{E}_{s}^{n}\right)$ and $\mathbf{h} \in L^{\infty}\left(\Gamma_{1}, \mathbb{R}^{n}\right)$.

LEMMA 18. Let $\widehat{f}: W^{n}(\Omega$, div $) \rightarrow \mathbb{R}$ be a linear functional, continuous in the topology (6.26), such that for every $\boldsymbol{\sigma}_{s} \in W^{n}(\Omega, \operatorname{div})$ with $\operatorname{div} \boldsymbol{\sigma}_{s}=\mathbf{0}$ in $\Omega$ we have $\widehat{f}\left(\boldsymbol{\sigma}_{s}\right)=0$. Then there exists $\widetilde{\mathbf{u}} \in L D(\Omega)$ such that for every $\boldsymbol{\sigma} \in W^{n}(\Omega, \operatorname{div})$,

$$
\widehat{f}(\boldsymbol{\sigma})=\int_{\Omega} \boldsymbol{\sigma}: \boldsymbol{\varepsilon}(\widetilde{\mathbf{u}}) d x-\int_{\operatorname{Fr} \Omega} \boldsymbol{\beta}_{B}(\boldsymbol{\sigma}) \cdot \gamma_{B}(\widetilde{\mathbf{u}}) d s,
$$

$\gamma_{B}(\widetilde{\mathbf{u}})=\mathbf{0}$ on $\Gamma_{0}, \gamma_{B}(\widetilde{\mathbf{u}}) \in L^{\infty}\left(\operatorname{Fr} \Omega, \mathbb{R}^{n}\right)$ and $\varepsilon(\widetilde{\mathbf{u}}) \in L^{\infty}\left(\Omega, \mathbf{E}_{s}^{n}\right)$.

Proof. The functional $\widehat{f}$ is continuous in the topology (6.26), so, by Theorem V.3.9 of [11], there exist $\mathbf{m} \in L^{\infty}\left(\Omega, \mathbf{E}_{s}^{n}\right)$ and $\widehat{\mathbf{u}} \in B D(\Omega)$ such that $\gamma_{B}(\widehat{\mathbf{u}})=\mathbf{0}$ on $\Gamma_{0}$, and $\widehat{f}(\boldsymbol{\sigma})=\int_{\Omega} \boldsymbol{\sigma}: \mathbf{m} d x-\int_{\mathrm{Fr} \Omega} \boldsymbol{\beta}_{B}(\boldsymbol{\sigma}) \cdot \gamma_{B}(\widehat{\mathbf{u}}) d s$ for all $\boldsymbol{\sigma} \in W^{n}(\Omega, \operatorname{div})$, since $L^{\infty}\left(\operatorname{Fr} \Omega, \mathbb{R}^{n}\right) \subset L^{1}\left(\operatorname{Fr} \Omega, \mathbb{R}^{n}\right)$ (cf. Proposition 1). For every $\boldsymbol{\sigma}_{1} \in W^{n}\left(\Omega_{1}\right.$, div) with $\operatorname{div} \boldsymbol{\sigma}_{1}=\mathbf{0}$ in $\Omega_{1}$ and $\boldsymbol{\sigma}_{1 \mid \bar{\Omega}} \in C\left(\bar{\Omega}, \mathbf{E}_{s}^{n}\right)$, we have

$$
\widehat{f}\left(\boldsymbol{\sigma}_{1 \mid \bar{\Omega}}\right)=\int_{\Omega} \boldsymbol{\sigma}_{1}: \mathbf{m} d x-\int_{\operatorname{Fr} \Omega} \boldsymbol{\sigma}_{1}:\left(\boldsymbol{\gamma}_{B}(\widehat{\mathbf{u}}) \otimes_{s} \boldsymbol{\nu}\right) d s=0 .
$$

Then by Proposition 1.1 and Theorem 1.3 of [23, Chapter 2] there exists $\widetilde{\mathbf{u}} \in L D(\Omega)$ such that equality (6.28) holds. 
Indeed, for all $\boldsymbol{\sigma}_{2} \in C_{0}^{1}\left(\Omega_{1}, \mathbf{E}_{s}^{n}\right)$ such that $\operatorname{div} \boldsymbol{\sigma}_{2}=\mathbf{0}$ in $\Omega_{1}$, we have $\int_{\Omega} \boldsymbol{\sigma}_{2}: \mathbf{m} d x-\int_{\operatorname{Fr} \Omega} \boldsymbol{\sigma}_{2}:\left(\boldsymbol{\gamma}_{B}(\widehat{\mathbf{u}}) \otimes_{s} \boldsymbol{\nu}\right) d s=0$. Then, by Proposition 1.1 of [23, Chapter 2], there exists $\widetilde{\mathbf{u}} \in D^{\prime}\left(\Omega_{1}, \mathbb{R}^{n}\right)$ such that for every $\boldsymbol{\sigma} \in C_{0}^{1}\left(\Omega_{1}, \mathbf{E}_{s}^{n}\right)$,

$$
\int_{\Omega_{1}} \boldsymbol{\sigma}: \varepsilon(\widetilde{\mathbf{u}})=\int_{\Omega} \boldsymbol{\sigma}: \mathbf{m} d x-\int_{\operatorname{Fr} \Omega} \boldsymbol{\sigma}:\left(\gamma_{B}(\widehat{\mathbf{u}}) \otimes_{s} \boldsymbol{\nu}\right) d s=\widehat{f}\left(\boldsymbol{\sigma}_{\mid \bar{\Omega}}\right),
$$

and

$$
\varepsilon(\widetilde{\mathbf{u}})= \begin{cases}\mathbf{m} d x & \text { in } \Omega, \\ -\left(\boldsymbol{\gamma}_{B}(\widehat{\mathbf{u}}) \otimes_{s} \boldsymbol{\nu}\right) d s & \text { on } \operatorname{Fr} \Omega, \\ \mathbf{0} & \text { in } \Omega_{1}-\bar{\Omega}\end{cases}
$$

(see [20]). For every $\boldsymbol{\sigma}_{3} \in C_{0}^{1}\left(\Omega_{1}, \mathbf{E}_{s}^{n}\right)$ such that $\boldsymbol{\sigma}_{3}=\mathbf{0}$ in $\bar{\Omega}$, we have $\int_{\Omega_{1}} \boldsymbol{\sigma}_{3}: \boldsymbol{\varepsilon}(\widetilde{\mathbf{u}})=\int_{\Omega} \boldsymbol{\sigma}_{3}: \mathbf{m} d x-\int_{\mathrm{Fr} \Omega} \boldsymbol{\sigma}_{3}:\left(\boldsymbol{\gamma}_{B}(\widehat{\mathbf{u}}) \otimes_{s} \boldsymbol{\nu}\right) d s=0$, therefore we can assume that $\widetilde{\mathbf{u}}_{\mid \Omega_{1}-\bar{\Omega}}=\mathbf{0}$. By Theorem 1.3 of [23, Chapter 2], $\widetilde{\mathbf{u}}_{\mid \Omega} \in L D(\Omega)$, because $\mathbf{m} \in L^{\infty}\left(\Omega, \mathbf{E}_{s}^{n}\right)$. Moreover, $\varepsilon\left(\widetilde{\mathbf{u}}_{\mid \Omega}\right) \in L^{\infty}\left(\Omega, \mathbf{E}_{s}^{n}\right)$ and $\gamma_{B}\left(\widetilde{\mathbf{u}}_{\mid \Omega}\right) \in$ $L^{\infty}\left(\operatorname{Fr} \Omega, \mathbb{R}^{n}\right)$.

Proposition 19. Let $r>0$ (in the definition of $T_{r}$ ) and $\mathbf{u}^{0}=\mathbf{0}$ on $\Gamma_{0}$. Then

$$
T_{r}^{*}(\mathbf{0})=\inf \left\{\widetilde{T}_{r}^{*}\left(\boldsymbol{\sigma}_{s}\right) \mid \boldsymbol{\sigma}_{s} \in W^{n}(\Omega, \operatorname{div}) \text { and } \operatorname{div} \boldsymbol{\sigma}_{s}=\mathbf{0} \text { in } \Omega\right\} .
$$

Proof. Step 1. Suppose there exists $\delta_{1}>0$ such that

$$
T_{r}^{*}(\mathbf{0})+\delta_{1}<\inf \left\{\widetilde{T}_{r}^{*}\left(\boldsymbol{\sigma}_{s}\right) \mid \boldsymbol{\sigma}_{s} \in W^{n}(\Omega, \operatorname{div}) \text { and } \operatorname{div} \boldsymbol{\sigma}_{s}=\mathbf{0} \text { in } \Omega\right\} .
$$

On account of Lemmas 14 and 17, it suffices to show that this assumption leads to a contradiction.

Let $\widetilde{T}_{r \mid L^{\infty}}: L^{\infty}\left(\Omega, \mathbf{E}_{s}^{n}\right) \times L^{\infty}\left(\Gamma_{1}, \mathbb{R}^{n}\right) \rightarrow \mathbb{R} \cup\{+\infty\}$ be the restriction of $\widetilde{T}_{r}$, given by $\widetilde{T}_{r \mid L^{\infty}}(\mathbf{w}, \mathbf{h})=\widetilde{T}_{r}\left(\mathbf{w},-\mathbf{h} \otimes_{s} \boldsymbol{\nu}\right)$ for $(\mathbf{w}, \mathbf{h}) \in L^{\infty}\left(\Omega, \mathbf{E}_{s}^{n}\right) \times$ $L^{\infty}\left(\Gamma_{1}, \mathbb{R}^{n}\right)$ (cf. (6.14)). Define the dual functional to $\widetilde{T}_{r \mid L^{\infty}}$ by

$$
\begin{array}{r}
\widetilde{T}_{r \mid L^{\infty}}^{*}(\boldsymbol{\sigma})=\sup \left\{\int_{\Omega} \boldsymbol{\sigma}: \mathbf{w} d x-\int_{\operatorname{Fr} \Omega} \boldsymbol{\beta}_{B}(\boldsymbol{\sigma}) \cdot \mathbf{h} d s-\widetilde{T}_{r \mid L^{\infty}}(\mathbf{w}, \mathbf{h}) \mid\right. \\
\left.\mathbf{w} \in L^{\infty}\left(\Omega, \mathbf{E}_{s}^{n}\right), \mathbf{h} \in L^{\infty}\left(\Gamma_{1}, \mathbb{R}^{n}\right)\right\}
\end{array}
$$

for $\boldsymbol{\sigma} \in W^{n}(\Omega, \operatorname{div})$. By (6.13) and (6.18) we obtain $\widetilde{T}_{r \mid L^{\infty}}^{*}(\boldsymbol{\sigma})=\widetilde{T}_{r}^{*}(\boldsymbol{\sigma})$ for every $\boldsymbol{\sigma} \in W^{n}(\Omega, \operatorname{div})$ (cf. Proposition 12). Therefore $\widetilde{T}_{r}^{*}: W^{n}(\Omega, \operatorname{div}) \rightarrow$ $\mathbb{R} \cup\{+\infty\}$ is l.s.c. in the topology (6.26).

Step 2. The linear space

$$
\mathcal{M}_{0} \equiv\left\{\boldsymbol{\sigma}_{s} \in W^{n}(\Omega, \operatorname{div}) \mid \operatorname{div} \boldsymbol{\sigma}_{s}=\mathbf{0} \text { in } \Omega\right\}
$$

is a closed subspace of $L^{\infty}\left(\Omega, \mathbf{E}_{s}^{n}\right)$ endowed with the topology $\sigma\left(L^{\infty}\left(\Omega, \mathbf{E}_{s}^{n}\right)\right.$, $\left.L^{\infty}\left(\Omega, \mathbf{E}_{s}^{n}\right)\right)$. Indeed, let $\left\{\boldsymbol{\sigma}_{k}\right\}_{k \in K} \subset W^{n}(\Omega, \operatorname{div})$ with $\operatorname{div} \boldsymbol{\sigma}_{k}=\mathbf{0}$ for every $k \in K$ be a net convergent to $\boldsymbol{\sigma}_{0} \in L^{\infty}\left(\Omega, \mathbf{E}_{s}^{n}\right)$ in the topology $\sigma\left(L^{\infty}\left(\Omega, \mathbf{E}_{s}^{n}\right)\right.$, 
$\left.L^{\infty}\left(\Omega, \mathbf{E}_{s}^{n}\right)\right)$, i.e. $\int_{\Omega}\left(\boldsymbol{\sigma}_{k}-\boldsymbol{\sigma}_{0}\right): \mathbf{z} d x \rightarrow 0$ for every $\mathbf{z} \in L^{\infty}\left(\Omega, \mathbf{E}_{s}^{n}\right)$. Then for every $\mathbf{u} \in C_{0}^{1}\left(\Omega, \mathbb{R}^{n}\right)$,

$$
0=-\int_{\Omega}\left(\operatorname{div} \boldsymbol{\sigma}_{k}\right) \cdot \mathbf{u} d x=\int_{\Omega} \boldsymbol{\sigma}_{k}: \varepsilon(u) d x \rightarrow \int_{\Omega} \boldsymbol{\sigma}_{0}: \boldsymbol{\varepsilon}(u) d x .
$$

Therefore $\operatorname{div} \boldsymbol{\sigma}_{0}=\mathbf{0}$ in the sense of distributions on $\Omega$.

Step 3. $\mathcal{M}_{0} \cap B_{L^{\infty}\left(\Omega, \mathbf{E}_{s}^{n}\right)}(0, \widehat{r})$ endowed with the topology (6.26) is compact, where $B_{L^{\infty}\left(\Omega, \mathbf{E}_{s}^{n}\right)}(0, \widehat{r})$ is the closed ball in $\left[L^{\infty}\left(\Omega, \mathbf{E}_{s}^{n}\right),\|\cdot\|_{L^{\infty}\left(\Omega, \mathbf{E}_{s}^{n}\right)}\right]$.

Indeed, let $\left\{\boldsymbol{\sigma}_{k}\right\}_{k \in K} \subset \mathcal{M}_{0} \cap B_{L^{\infty}\left(\Omega, \mathbf{E}_{s}^{n}\right)}(0, \widehat{r})$ be a net. Then there exists a finer net $\left\{\boldsymbol{\sigma}_{k_{t}}\right\}_{t \in T}$ and $\boldsymbol{\sigma}_{0} \in \mathcal{M}_{0} \cap B_{L^{\infty}\left(\Omega, \mathbf{E}_{s}^{n}\right)}(0, \widehat{r})$ such that

$$
\int_{\Omega}\left(\boldsymbol{\sigma}_{k_{t}}-\boldsymbol{\sigma}_{0}\right): \mathbf{z} d x \rightarrow 0 \quad \text { for every } \mathbf{z} \in L^{\infty}\left(\Omega, \mathbf{E}_{s}^{n}\right),
$$

because $\sigma\left(L^{\infty}\left(\Omega, \mathbf{E}_{s}^{n}\right), L^{\infty}\left(\Omega, \mathbf{E}_{s}^{n}\right)\right)$ is weaker than $\sigma\left(L^{\infty}\left(\Omega, \mathbf{E}_{s}^{n}\right), L^{1}\left(\Omega, \mathbf{E}_{s}^{n}\right)\right)$, and $\left[L^{\infty}\left(\Omega, \mathbf{E}_{s}^{n}\right), \sigma\left(L^{\infty}\left(\Omega, \mathbf{E}_{s}^{n}\right), L^{\infty}\left(\Omega, \mathbf{E}_{s}^{n}\right)\right)\right]$ is a Hausdorff topological space (cf. [13, Corollary 3.1.14]). The trace $\boldsymbol{\beta}_{B}$ is a continuous linear map from $\left[W^{n}(\Omega, \operatorname{div}),\|\cdot\|_{W^{n}(\Omega, \operatorname{div})}\right]$ into $\left[L^{\infty}\left(\operatorname{Fr} \Omega, \mathbb{R}^{n}\right),\|\cdot\|_{L^{\infty}}\right]$. Hence the net $\left\{\boldsymbol{\beta}_{B}\left(\boldsymbol{\sigma}_{k_{t}}\right)\right\}_{t \in T}$ is bounded in $\left[L^{\infty}\left(\operatorname{Fr} \Omega, \mathbb{R}^{n}\right),\|\cdot\|_{L^{\infty}}\right]$, since $\left\{\boldsymbol{\sigma}_{k_{t}}\right\}_{t \in T} \subset \mathcal{M}_{0}$. Therefore there exists a finer net $\left\{\boldsymbol{\sigma}_{k_{t_{p}}}\right\}_{p \in P}$ and $\widetilde{\varrho} \in L^{\infty}\left(\mathrm{Fr} \Omega, \mathbb{R}^{n}\right)$ such that $\int_{\mathrm{Fr} \Omega}\left(\boldsymbol{\beta}_{B}\left(\boldsymbol{\sigma}_{k_{t}}\right)-\widetilde{\varrho}\right) \cdot \widetilde{\mathbf{h}} d s \rightarrow 0$ for every $\widetilde{\mathbf{h}} \in L^{1}\left(\operatorname{Fr} \Omega, \mathbb{R}^{n}\right)$. Moreover, by $(2.11)$ we obtain

$$
\int_{\Omega}\left(\boldsymbol{\sigma}_{k_{t_{p}}}\right): \boldsymbol{\varepsilon}(\mathbf{u}) d x=\int_{\operatorname{Fr} \Omega} \boldsymbol{\beta}_{B}\left(\boldsymbol{\sigma}_{k_{t_{p}}}\right) \cdot \boldsymbol{\gamma}_{B}(\mathbf{u}) d s \quad \forall \mathbf{u} \in W^{n}(\Omega, \operatorname{div}),
$$

and so

$$
\int_{\operatorname{Fr} \Omega} \boldsymbol{\beta}_{B}\left(\boldsymbol{\sigma}_{0}\right) \cdot \gamma_{B}(\mathbf{u}) d s=\int_{\Omega} \boldsymbol{\sigma}_{0}: \boldsymbol{\varepsilon}(\mathbf{u}) d x=\int_{\operatorname{Fr} \Omega} \widetilde{\boldsymbol{\varrho}} \cdot \boldsymbol{\gamma}_{B}(\mathbf{u}) d s .
$$

Therefore $\boldsymbol{\beta}_{B}\left(\boldsymbol{\sigma}_{0}\right)=\widetilde{\varrho}$.

Step 4. By (5.3), (5.4), (4.8) and Assumption 5, $\widetilde{T}_{r}^{*}(\mathbf{0})=\inf \left\{\widetilde{T}_{r}^{*}\left(\boldsymbol{\sigma}_{s}\right) \mid\right.$ $\boldsymbol{\sigma}_{s} \in W^{n}(\Omega, \operatorname{div})$ and $\operatorname{div} \boldsymbol{\sigma}_{s}=\mathbf{0}$ in $\left.\Omega\right\}$. By the Hahn-Banach theorem, for every $m \in \mathbb{N}$, there exists an affine functional

$$
W^{n}(\Omega, \operatorname{div}) \ni \boldsymbol{\sigma} \mapsto \widetilde{f}_{m}(\boldsymbol{\sigma})+\widetilde{a}_{m} \in \mathbb{R}
$$

such that

$$
\widetilde{f}_{m}(\boldsymbol{\sigma})+\widetilde{a}_{m}<\widetilde{T}_{r}^{*}(\boldsymbol{\sigma}) \text { and } \widetilde{f}_{m}(\widetilde{\boldsymbol{\sigma}})+\widetilde{a}_{m}>\widetilde{T}_{r}^{*}(\mathbf{0})-\delta_{1} / 2^{m}
$$

for all $\boldsymbol{\sigma} \in W^{n}\left(\Omega\right.$, div) and all $\tilde{\boldsymbol{\sigma}} \in \mathcal{M}_{0} \cap B_{L^{\infty}\left(\Omega, \mathbf{E}_{s}^{n}\right)}\left(0, \widehat{r} 2^{m}\right)$, where $\tilde{f}_{m}$ is continuous in the topology (6.26). Indeed, $\mathcal{M}_{0} \cap B_{L^{\infty}\left(\Omega, \mathbf{E}_{s}^{n}\right)}\left(0, \widehat{r} 2^{m}\right)$ is compact and $\widetilde{T}_{r}^{*}$ is l.s.c. in this topology. By (6.17) and Assumption 5, $\widetilde{T}_{r}^{*}(\mathbf{0})<\infty$. 
Step 5. Let $f_{0}: W^{n}(\Omega$, div $) \rightarrow \mathbb{R}$ be given by

$$
f_{0}(\boldsymbol{\sigma})=\int_{\Omega} \boldsymbol{\sigma}: \mathbf{m}^{0} d x+\int_{\Gamma_{1}} \boldsymbol{\beta}_{B}(\boldsymbol{\sigma}) \cdot \mathbf{h}^{0} d s
$$

where $\left(\mathbf{m}^{0}, \mathbf{h}^{0}\right) \in L^{\infty}\left(\Omega, \mathbf{E}_{s}^{n}\right) \times L^{\infty}\left(\Gamma_{1}, \mathbb{R}^{n}\right)$, and assume that

$$
f_{0}(\boldsymbol{\sigma})+\widetilde{T}_{r}^{*}(\mathbf{0}) \leq \widetilde{T}_{r}^{*}(\boldsymbol{\sigma}), \quad \forall \boldsymbol{\sigma} \in W^{n}(\Omega, \operatorname{div}) .
$$

Define

$$
k_{e} \equiv\left(\sum_{i, j, k, l=1}^{n}\left\|a_{i j k l}\right\|_{L^{\infty}}\right)\left\{\sum_{i, j=1}^{n}\left[\left\|q_{i j}\right\|_{L^{\infty}}+\left\|\left(\boldsymbol{\sigma}_{L}\right)_{i j}\right\|_{L^{\infty}}+1\right]\right\}^{2} .
$$

Then we obtain $k_{e} \geq\left\|\mathbf{m}^{0}\right\|_{L^{\infty}\left(\Omega, \mathbf{E}_{s}^{n}\right)}($ see $(3.3),(6.17)$ and Assumption 5). Moreover, there exists a constant $c_{s}$, which depends only on $\Omega$, such that

$$
k_{e} \geq c_{s}\left\|\mathbf{h}^{0}\right\|_{L^{\infty}\left(\Gamma_{1}, \mathbb{R}^{n}\right)} .
$$

The field $\left[q_{i j}\right] \in L^{\infty}\left(\Omega, \mathbf{E}_{s}^{n}\right)$ was introduced in Assumption 3 .

Indeed, let $A_{t} \subset \Omega$ be a measurable set such that $d x\left(A_{t}\right)>0,\left\|\mathbf{m}^{0}(x)\right\|_{\mathbf{E}_{s}^{n}}$ $>k_{e}+\delta_{s}$ for $d x$-a.e. $x \in A_{t}$ (where $\delta_{s}>0$ ), and there exists $\mathbf{m}^{c} \in \mathbf{E}_{s}^{n}$ such that $\left\|\mathbf{m}^{0}(x): \mathbf{m}^{c}\right\|_{\mathbf{E}_{s}^{n}}>\left(k_{e}+\delta_{s} / 2\right)\left\|\mathbf{m}^{c}\right\|_{\mathbf{E}_{s}^{n}}$ for $d x$-a.e. $x \in A_{t}$. Since the Lebesgue measure $d x$ is regular, for every $k \in \mathbb{N}$ there exists a closed set $A_{c}^{k}$ and an open set $A_{o}^{k}$ such that $A_{c}^{k} \subset A_{t} \subset A_{o}^{k} \subset \Omega$ and $d x\left(A_{o}^{k}-A_{c}^{k}\right)<1 / k$. By Urysohn's Lemma [13, Theorem 1.5.10], for every $k \in \mathbb{N}$, there exists a continuous function $\varphi_{k} \in C\left(\mathbb{R}^{n}, \mathbb{R}\right)$ such that $\varphi_{k \mid A_{c}^{k}}=1, \varphi_{k \mid \mathbb{R}^{n}-A_{o}^{k}}=0$ and $0 \leq \varphi_{k}(x) \leq 1$ for every $x \in \mathbb{R}^{n}$. Then $\varphi_{k \mid \bar{\Omega}} \in C_{0}(\bar{\Omega}, \mathbb{R})$. Moreover, there exists $k_{0} \in \mathbb{N}$ such that for every $\tilde{k}>k_{0}$,

$$
\int_{\Omega} \delta_{0} \varphi_{\widetilde{k}}(x) \frac{\mathbf{m}^{c}}{\left\|\mathbf{m}^{c}\right\|_{\mathbf{E}_{s}^{n}}}: \mathbf{m}^{0}(x) d x+\widetilde{T}_{r}^{*}(\mathbf{0})>\widetilde{T}_{r}^{*}\left(\delta_{0} \varphi_{\widetilde{k}}(x) \frac{\mathbf{m}^{c}}{\left\|\mathbf{m}^{c}\right\|_{\mathbf{E}_{s}^{n}}}\right),
$$

where $\delta_{0}<1$ is given in Assumption 5. The space $C_{0}^{1}(\bar{\Omega}, \mathbb{R})$ is dense in $\left[C_{0}(\bar{\Omega}, \mathbb{R}),\|\cdot\|_{C}\right]$. Hence there exists $\varphi_{C^{1}} \in C_{0}^{1}(\bar{\Omega}, \mathbb{R})$ which satisfies $(6.45)$ with $\varphi_{\widetilde{k}}$ replaced by $\varphi_{C^{1}}$. Therefore $k_{e} \geq\left\|\mathbf{m}^{0}\right\|_{L^{\infty}\left(\Omega, \mathbf{E}_{s}^{n}\right)}$. The equality (6.44) is obtained directly, since $\boldsymbol{\beta}_{B}: W^{n}(\Omega, \operatorname{div}) \rightarrow L^{\infty}\left(\operatorname{Fr} \Omega, \mathbb{R}^{n}\right)$ is a surjection, i.e. for every $\mathbf{p} \in \boldsymbol{\beta}_{B}\left(W^{n}(\Omega\right.$, div $\left.)\right)$ there exists $\overline{\mathbf{p}} \in W^{n}(\Omega$, div $)$ such that $\|\overline{\mathbf{p}}\|_{W^{n}(\Omega, \operatorname{div})} \leq c_{s}\|\mathbf{p}\|_{L^{\infty}\left(\operatorname{Fr} \Omega, \mathbb{R}^{n}\right)}$ and $\mathbf{p}=\boldsymbol{\beta}_{B}(\overline{\mathbf{p}})$ (cf. [11, Theorem II.2.1]).

Step 6. For every $m \in \mathbb{N}$ the functional $\tilde{f}_{m}$ defined in Step 4 is continuous in (6.26). Hence there exist $\widetilde{\mathbf{w}}_{m} \in L^{\infty}\left(\Omega, \mathbf{E}_{s}^{n}\right)$ and $\widetilde{\mathbf{h}}_{m} \in L^{\infty}\left(\Gamma_{1}, \mathbb{R}^{n}\right)$ such that

$$
\widetilde{f}_{m}(\boldsymbol{\sigma})=\int_{\Omega} \boldsymbol{\sigma}: \widetilde{\mathbf{w}}_{m} d x+\int_{\Gamma_{1}} \boldsymbol{\beta}_{B}(\boldsymbol{\sigma}) \cdot \widetilde{\mathbf{h}}_{m} d s, \quad \forall \boldsymbol{\sigma} \in W^{n}(\Omega, \operatorname{div})
$$

(see $[11$, Theorem V.3.9]). Because of (6.40), for every $m \in \mathbb{N}$ there exist 
measurable sets $A_{m} \subset \Omega$ and $\widetilde{A}_{m} \subset \Gamma_{1}$ such that

$$
d x\left(A_{m}\right)<\frac{\delta_{1}}{5 k_{e} \delta_{0} 2^{m}}, \quad d s\left(\widetilde{A}_{m}\right)<\frac{\delta_{1}}{5 k_{e} r 2^{m}},
$$

where $k_{e}$ is defined in (6.43), $\delta_{0}$ is given in Assumption $5, r$ is given in (5.1), and

$$
\left\|\widetilde{\mathbf{w}}_{m}\right\|_{L^{\infty}\left(\Omega-A_{m}, \mathbf{E}_{s}^{n}\right)}<10 k_{e}, \quad c_{s}\left\|\widetilde{\mathbf{h}}_{m}\right\|_{L^{\infty}\left(\Gamma_{1}-\widetilde{A}_{m}, \mathbb{R}^{n}\right)}<10 k_{e} .
$$

We have

$$
d x\left(\bigcup_{m=m_{1}}^{\infty} A_{m}\right)<\frac{\delta_{1}}{5 k_{e} \delta_{0} 2^{m_{1}-1}}, \quad d s\left(\bigcup_{m=m_{1}}^{\infty} \widetilde{A}_{m}\right)<\frac{\delta_{1}}{5 k_{e} r 2^{m_{1}-1}}
$$

for every $m_{1} \geq 1$. Thus there exist $\widetilde{m}_{1} \in \mathbb{N}$, a subsequence $\left\{\left(\widetilde{\mathbf{w}}_{m_{k}}, \widetilde{\mathbf{h}}_{m_{k}}\right)\right\}_{k \in \mathbb{N}}$ of $\left\{\left(\widetilde{\mathbf{w}}_{m}, \widetilde{\mathbf{h}}_{m}\right)\right\}_{m \geq \widetilde{m}_{1}}$ and $\left(\widetilde{\mathbf{w}}_{0}^{\widetilde{m}_{1}}, \widetilde{\mathbf{h}}_{0}^{\widetilde{m}_{1}}\right) \in L^{\infty}\left(\Omega-\bigcup_{m=\widetilde{m}_{1}}^{\infty} A_{m}, \mathbf{E}_{s}^{n}\right) \times L^{\infty}\left(\Gamma_{1}-\right.$ $\left.\bigcup_{m=\tilde{m}_{1}}^{\infty} \widetilde{A}_{m}, \mathbb{R}^{n}\right)$ such that

$$
\begin{gathered}
\widetilde{\mathbf{w}}_{m_{k}} \rightarrow \widetilde{\mathbf{w}}_{0}^{\widetilde{m}_{1}} \quad \text { weak }^{*} \text { in } L^{\infty}\left(\Omega-\bigcup_{m=\widetilde{m}_{1}}^{\infty} A_{m}, \mathbf{E}_{s}^{n}\right), \\
\widetilde{\mathbf{h}}_{m_{k}} \rightarrow \widetilde{\mathbf{h}}_{0}^{\widetilde{m}_{1}} \quad \text { weak }^{*} \text { in } L^{\infty}\left(\Gamma_{1}-\bigcup_{m=\widetilde{m}_{1}}^{\infty} \widetilde{A}_{m}, \mathbb{R}^{n}\right) .
\end{gathered}
$$

Let

$$
\left\{\left(\widetilde{\mathbf{w}}_{m_{k}}, \widetilde{\mathbf{h}}_{m_{k}}\right)\right\}_{k \in \mathbb{N}}^{m_{k} \geq \widetilde{m}_{2}}=\left\{\left(\widetilde{\mathbf{w}}_{m_{k}}, \widetilde{\mathbf{h}}_{m_{k}}\right)\right\}_{k \in \mathbb{N}} \cap\left\{\left(\widetilde{\mathbf{w}}_{m}, \widetilde{\mathbf{h}}_{m}\right)\right\}_{m \geq \tilde{m}_{2}} .
$$

If $\widetilde{m}_{2}>\widetilde{m}_{1}$, then there exists

$$
\left(\widetilde{\mathbf{w}}_{0}^{\widetilde{m}_{2}}, \widetilde{\mathbf{h}}_{0}^{\widetilde{m}_{2}}\right) \in L^{\infty}\left(\Omega-\bigcup_{m=\widetilde{m}_{2}}^{\infty} A_{m}, \mathbf{E}_{s}^{n}\right) \times L^{\infty}\left(\Gamma_{1}-\bigcup_{m=\widetilde{m}_{2}}^{\infty} \widetilde{A}_{m}, \mathbb{R}^{n}\right)
$$

such that

$$
\begin{aligned}
\left(\widetilde{\mathbf{w}}_{m_{k}}, \widetilde{\mathbf{h}}_{m_{k}}\right) & \rightarrow\left(\widetilde{\mathbf{w}}_{0}^{\tilde{m}_{2}}, \widetilde{\mathbf{h}}_{0}^{\tilde{m}_{2}}\right) \\
& \text { weak }^{*} \text { in } L^{\infty}\left(\Omega-\bigcup_{m=\widetilde{m}_{2}}^{\infty} A_{m}\right) \times L^{\infty}\left(\Gamma_{1}-\bigcup_{m=\widetilde{m}_{2}}^{\infty} \widetilde{A}_{m}\right),
\end{aligned}
$$

$\widetilde{\mathbf{w}}_{0}^{\widetilde{m}_{2}}(x)=\widetilde{\mathbf{w}}_{0}^{\widetilde{m}_{1}}(x)$ for $d x$-a.e. $x \in \Omega-\bigcup_{m=\widetilde{m}_{1}}^{\infty} A_{m}$ and $\widetilde{\mathbf{h}}_{0}^{\widetilde{m}_{2}}(x)=\widetilde{\mathbf{h}}_{0}^{\widetilde{m}_{1}}(x)$ for ds-a.e. $x \in \Gamma_{1}-\bigcup_{m=\widetilde{m}_{1}}^{\infty} \widetilde{A}_{m}$. Letting $\widetilde{m}_{k} \rightarrow \infty$, we obtain

$$
\begin{aligned}
& \widetilde{\mathbf{w}}_{0}=\widetilde{\mathbf{w}}_{0 \mid \Omega-\cup_{m=\widetilde{m}_{1}} A_{m}}^{\widetilde{m}_{1}}+\sum_{k=2}^{\infty} \widetilde{\mathbf{w}}_{0 \mid A_{\widetilde{m}_{k-1}}-\cup_{m=\widetilde{m}_{k}}^{\infty} A_{m},}^{\widetilde{m}_{k}} \widetilde{\mathbf{h}}_{0}=\widetilde{\mathbf{h}}_{0 \mid \Gamma_{1}-\cup_{m=\widetilde{m}_{1}}^{\infty} \widetilde{A}_{m}+\sum_{k=2}^{\infty} \widetilde{\mathbf{h}}_{0 \mid \widetilde{A}_{\widetilde{m}_{k-1}} \widetilde{m}_{k}} \cup_{m=\widetilde{m}_{k}}^{\infty} \widetilde{A}_{m},},
\end{aligned}
$$

where $\widetilde{\mathbf{w}}_{0} \in L^{\infty}\left(\Omega, \mathbf{E}_{s}^{n}\right), \widetilde{\mathbf{h}}_{0} \in L^{\infty}\left(\Gamma_{1}, \mathbb{R}^{n}\right),\left\|\widetilde{\mathbf{w}}_{0}\right\|_{L^{\infty}} \leq 10 k_{e},\left\|\widetilde{\mathbf{h}}_{0}\right\|_{L^{\infty}} \leq 10 k_{e}$. 
Step 7. Let $\mathbf{p} \in L^{1}\left(\Omega, \mathbf{E}_{s}^{n}\right)$ and suppose there exists $\widetilde{m}_{k_{0}}$ such that

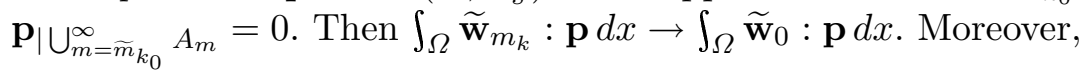

$$
\int_{A_{m_{k}}} \widetilde{\mathbf{w}}_{m_{k}}: \mathbf{t} d x \leq \frac{\delta_{1}}{\delta_{0} 2^{m_{k}}}\|\mathbf{t}\|_{L^{\infty}}+10 k_{e}\|\mathbf{t}\|_{L^{\infty}} d x\left(A_{m_{k}}\right)
$$

for all $\mathbf{t} \in L^{\infty}\left(\Omega, \mathbf{E}_{s}^{n}\right)$ and $k \in \mathbb{N}$ (cf. (6.50)). We obtain (6.57) from the inequalities

$$
\widetilde{T}_{r}^{*}(\boldsymbol{\sigma})>\widetilde{f}_{m_{k}}(\boldsymbol{\sigma})+\widetilde{a}_{m_{k}}, \quad \widetilde{a}_{m_{k}}>\widetilde{T}_{r}^{*}(\mathbf{0})-\frac{\delta_{1}}{2^{m_{k}}} \quad \forall \boldsymbol{\sigma} \in W^{n}(\Omega, \operatorname{div})
$$

(cf. (6.40) and Assumption 5). Moreover, we have

$$
\begin{aligned}
\int_{\left(\cup_{m>m_{k}}^{\infty}\right.}{\widetilde{A_{m}}}_{A_{m_{k}}-A_{m_{k}}}: \mathbf{t} d x & \leq 10 k_{e}\|\mathbf{t}\|_{L^{\infty}} d x\left(\bigcup_{m>m_{k}}^{\infty} A_{m}\right) \\
& \leq \frac{2 \delta_{1}}{\delta_{0} 2^{m_{k}}}\|\mathbf{t}\|_{L^{\infty}}
\end{aligned}
$$

(cf. (6.49)). Then for every $\mathbf{t} \in L^{\infty}\left(\Omega, \mathbf{E}_{s}^{n}\right)$ and every $\widetilde{\delta}$, there exists $\widetilde{k} \in \mathbb{N}$ such that for every $k>\widetilde{k},\left|\int_{\Omega}\left(\widetilde{\mathbf{w}}_{m_{k}}-\widetilde{\mathbf{w}}_{0}\right): \mathbf{t} d x\right| \leq \widetilde{\delta}$, since $\left\|\widetilde{\mathbf{w}}_{0}\right\|_{L^{\infty}} \leq 10 k_{e}$. Therefore $\widetilde{\mathbf{w}}_{m_{k}} \rightarrow \widetilde{\mathbf{w}}_{0}$ in the topology $\sigma\left(L^{\infty}\left(\Omega, \mathbf{E}_{s}^{n}\right), L^{\infty}\left(\Omega, \mathbf{E}_{s}^{n}\right)\right)$. Similarly, we prove that $\widetilde{\mathbf{h}}_{m_{k}} \rightarrow \widetilde{\mathbf{h}}_{0}$ in the topology $\sigma\left(L^{\infty}\left(\Gamma_{1}, \mathbb{R}^{n}\right), L^{\infty}\left(\Gamma_{1}, \mathbb{R}^{n}\right)\right)$.

Step 8. Because of (6.39) and (6.40) the functional

$$
W^{n}(\Omega, \operatorname{div}) \ni \boldsymbol{\sigma} \mapsto \widetilde{f}_{0}(\boldsymbol{\sigma})=\int_{\Omega} \boldsymbol{\sigma}: \widetilde{\mathbf{w}}_{0} d x+\int_{\Gamma_{1}} \boldsymbol{\beta}_{B}(\boldsymbol{\sigma}) \cdot \widetilde{\mathbf{h}}_{0} d s \in \mathbb{R}
$$

satisfies

$$
\widetilde{f}_{0}(\boldsymbol{\sigma})+\widetilde{T}_{r}^{*}(\mathbf{0})<\widetilde{T}_{r}^{*}(\boldsymbol{\sigma}), \quad \widetilde{f}_{0}\left(\boldsymbol{\sigma}_{s}\right) \geq 0
$$

for all $\boldsymbol{\sigma} \in W^{n}\left(\Omega\right.$, div) and all $\boldsymbol{\sigma}_{s} \in \mathcal{M}_{0}$ (cf. (6.33) and (6.35)). Then $\widetilde{f}_{0}\left(\boldsymbol{\sigma}_{s}\right)=0$ for every $\boldsymbol{\sigma}_{s} \in \mathcal{M}_{0}$. By Lemma 18 , there exists $\widetilde{\mathbf{u}} \in L D(\Omega)$ such that for every $\boldsymbol{\sigma} \in W^{n}(\Omega, \operatorname{div})$,

$$
\widetilde{f}_{0}(\boldsymbol{\sigma})=\int_{\Omega} \boldsymbol{\sigma}: \boldsymbol{\varepsilon}(\widetilde{\mathbf{u}}) d x-\int_{\operatorname{Fr} \Omega} \boldsymbol{\beta}_{B}(\boldsymbol{\sigma}) \cdot \gamma_{B}(\widetilde{\mathbf{u}}) d s
$$

$\gamma_{B}(\widetilde{\mathbf{u}})=0$ on $\Gamma_{0}, \gamma_{B}(\widetilde{\mathbf{u}}) \in L^{\infty}\left(\operatorname{Fr} \Omega, \mathbb{R}^{n}\right)$ and $\varepsilon(\widetilde{\mathbf{u}}) \in L^{\infty}\left(\Omega, \mathbf{E}_{s}^{n}\right)$.

Step 9. We say that a net $\left\{\boldsymbol{\sigma}_{k}\right\}_{k \in K} \subset C_{\operatorname{div}}\left(\bar{\Omega}, \mathbf{E}_{s}^{n}\right)$ converges to $\widehat{\boldsymbol{\sigma}} \in$ $C_{\text {div }}\left(\bar{\Omega}, \mathbf{E}_{s}^{n}\right)$ in the topology $\sigma\left(C_{\operatorname{div}}\left(\bar{\Omega}, \mathbf{E}_{s}^{n}\right), L D(\Omega)\right)$ if

$$
\int_{\Omega}\left(\boldsymbol{\sigma}_{k}-\widehat{\boldsymbol{\sigma}}\right): \boldsymbol{\varepsilon}(\mathbf{u}) d x-\int_{\operatorname{Fr} \Omega}\left(\boldsymbol{\sigma}_{k}-\widehat{\boldsymbol{\sigma}}\right):\left(\boldsymbol{\gamma}_{B}(\mathbf{u}) \otimes_{s} \boldsymbol{\nu}\right) d s \rightarrow 0
$$

for every $\mathbf{u} \in L D(\Omega)$ such that $\gamma_{B}(\mathbf{u})=\mathbf{0}$ on $\Gamma_{0}$. The l.s.c. regularization of $\widetilde{T}_{r}^{*}$ in the topology $\sigma\left(C_{\operatorname{div}}\left(\bar{\Omega}, \mathbf{E}_{s}^{n}\right), L D(\Omega)\right.$ ) (denoted by $\left.\mathrm{cl}_{\sigma(C, L D)} \widetilde{T}_{r}^{*}\right)$ is 
given by

$$
\operatorname{cl}_{\sigma(C, L D)} \widetilde{T}_{r}^{*}(\boldsymbol{\sigma})=\sup \left\{\int_{\Omega} \boldsymbol{\sigma}: \boldsymbol{\varepsilon}(\mathbf{u})_{\mid \Omega} d x-\int_{\operatorname{Fr} \Omega} \boldsymbol{\beta}_{B}(\boldsymbol{\sigma}) \gamma_{B}^{I}(\mathbf{u}) d s\right.
$$

$$
\begin{aligned}
& \left.-\widetilde{T}_{r}^{* \#}\left(\varepsilon(\mathbf{u})_{\mid \bar{\Omega}}\right) \mid \mathbf{u} \in B D\left(\Omega_{1}\right), \mathbf{u}_{\mid \Omega} \in L D(\Omega), \mathbf{u}_{\mid \Omega_{1}-\bar{\Omega}}=\mathbf{0}, \gamma_{B}^{I}(\mathbf{u})=\mathbf{0} \text { on } \Gamma_{0}\right\} \\
& =\sup \left\{\int_{\Omega} \boldsymbol{\sigma}: \boldsymbol{\varepsilon}(\mathbf{u})_{\mid \Omega} d x-\int_{\operatorname{Fr} \Omega} \boldsymbol{\beta}_{B}(\boldsymbol{\sigma}) \gamma_{B}^{I}(\mathbf{u}) d s-T_{r}\left(\boldsymbol{\varepsilon}(\mathbf{u})_{\mid \bar{\Omega}}\right) \mid\right.
\end{aligned}
$$

$$
\left.\mathbf{u} \in B D\left(\Omega_{1}\right), \mathbf{u}_{\mid \Omega} \in L D(\Omega), \mathbf{u}_{\mid \Omega_{1}-\bar{\Omega}}=\mathbf{0}, \gamma_{B}^{I}(\mathbf{u})=\mathbf{0} \text { on } \Gamma_{0}\right\}=T_{r}^{*}(\boldsymbol{\sigma}),
$$

for every $\boldsymbol{\sigma} \in C_{\text {div }}\left(\bar{\Omega}, \mathbf{E}_{s}^{n}\right.$ ) (cf. Proposition 15 and Lemma 16). From (6.33), (6.61), (6.62) and (6.64) we obtain a contradiction.

Proposition 20. Let $\mathbf{u}^{0}=\mathbf{0}$ on $\Gamma_{0}$. For every $r>0$,

$$
\inf \left\{T_{r}(\mathbf{M}) \mid \mathbf{M} \in \mathbf{Y}^{1}(\bar{\Omega})\right\}=\inf \left\{\widetilde{T}_{r}(\mathbf{M}) \mid \mathbf{M} \in \widetilde{\mathbf{Y}}^{1}(\bar{\Omega})\right\} .
$$

Proof. By (6.15), (6.17), (4.8), (5.3), (5.4), Assumption 5, (6.17), Proposition 19 and (6.10), we have

$$
\begin{aligned}
& \sup \left\{-\widetilde{T}_{r}(\mathbf{M}) \mid \mathbf{M} \in \widetilde{\mathbf{Y}}^{1}(\bar{\Omega})\right\}=\widetilde{T}_{r}^{*}(\mathbf{0}) \\
& \quad=-\left(P_{\lambda, r}^{*}\right)\left(\boldsymbol{\sigma}_{L}\right)=\inf \left\{-\left(P_{\lambda, r}^{*}\right)(\boldsymbol{\sigma}) \mid \boldsymbol{\sigma} \in W^{n}(\Omega, \operatorname{div})\right\} \\
& \quad=\inf \left\{\widetilde{T}_{r}^{*}\left(\boldsymbol{\sigma}_{s}\right) \mid \boldsymbol{\sigma}_{s} \in W^{n}(\Omega, \operatorname{div}), \operatorname{div} \boldsymbol{\sigma}_{s}=\mathbf{0}\right\}=T_{r}^{*}(\mathbf{0}) \\
& \quad=\sup \left\{-T_{r}(\mathbf{M}) \mid \mathbf{M} \in \mathbf{Y}^{1}(\bar{\Omega})\right\} .
\end{aligned}
$$

Let

$$
\operatorname{cl}_{\sigma\left(\mathbf{Y}^{1}(\bar{\Omega}), C_{\mathrm{div}}\left(\bar{\Omega}, \mathbf{E}_{s}^{n}\right)\right)} T_{r} \quad\left(\operatorname{resp} \cdot \operatorname{cl}_{\sigma\left(\widetilde{\mathbf{Y}}^{1}(\bar{\Omega}), C_{\mathrm{div}}\left(\bar{\Omega}, \mathbf{E}_{s}^{n}\right)\right)} \widetilde{T}_{r}\right)
$$

denote the largest l.s.c. minorant of $T_{r}$ in $\sigma\left(\mathbf{Y}^{1}(\bar{\Omega}), C_{\operatorname{div}}\left(\bar{\Omega}, \mathbf{E}_{s}^{n}\right)\right.$ ) (respectively, the largest l.s.c. minorant of $\widetilde{T}_{r}$ in $\left.\sigma\left(\widetilde{\mathbf{Y}}^{1}(\bar{\Omega}), C_{\operatorname{div}}\left(\bar{\Omega}, \mathbf{E}_{s}^{n}\right)\right)\right)$, i.e. $(6.67)$ stands for the l.s.c. regularizations of $T_{r}$ and $\widetilde{T}_{r}$ in the above mentioned topologies.

Because $\mathbf{0} \in C_{\operatorname{div}}\left(\bar{\Omega}, \mathbf{E}_{s}^{n}\right)$ and from Proposition 20 we get

$$
\begin{aligned}
& \inf \left\{\mathrm{cl}_{\sigma\left(\mathbf{Y}^{1}(\bar{\Omega}), C_{\operatorname{div}}\left(\bar{\Omega}, \mathbf{E}_{s}^{n}\right)\right)} T_{r}(\mathbf{M}) \mid \mathbf{M} \in \mathbf{Y}^{1}(\bar{\Omega})\right\} \\
& =\inf \left\{\mathrm{cl}_{\sigma\left(\widetilde{\mathbf{Y}}^{1}(\bar{\Omega}), C_{\operatorname{div}}\left(\bar{\Omega}, \mathbf{E}_{s}^{n}\right)\right)} \widetilde{T}_{r}(\mathbf{M}) \mid \mathbf{M} \in \widetilde{\mathbf{Y}}^{1}(\bar{\Omega})\right\} .
\end{aligned}
$$

TheOrem 21. Let $r>0$ and $\mathbf{u}^{0}=\mathbf{0}$ on $\Gamma_{0}$. Let Assumptions 3,5 and 6 hold. If $T_{r}$ is a coercive function, then by (6.20) and Proposition 15 the functional $T_{r}^{* *}$ is given by $(6.20)$, since $T_{r}^{* *}=\widetilde{T}_{r}^{* \#}$. Moreover, every minimum point $\boldsymbol{\varepsilon}(\widehat{\mathbf{u}})_{\mid \bar{\Omega}} \in \mathbf{Y}^{1}(\bar{\Omega})$ of $T_{r}^{* *}$ is given by a function $\widehat{\mathbf{u}} \in B D\left(\Omega_{1}\right)$ such that $\widehat{\mathbf{u}}_{\mid \Omega} \in L D(\Omega), \widehat{\mathbf{u}}_{\mid \Omega_{1}-\bar{\Omega}}=0$ and $\gamma_{B}(\widehat{\mathbf{u}})=\mathbf{0}$ on $\Gamma_{0}$.

Proof. Step 1. Let $\varepsilon\left(\widehat{\mathbf{u}}_{1}\right)_{\mid \bar{\Omega}} \in \mathbf{Y}^{1}(\bar{\Omega})$ be a minimum point of $T_{r}^{* *}$. By (6.10), (6.19) and Proposition 15 the functional $T_{r}^{* *}$ is the l.s.c. regularization 
of $T_{r}$ in the topology $\sigma\left(\mathbf{Y}^{1}(\bar{\Omega}), C_{\operatorname{div}}\left(\bar{\Omega}, \mathbf{E}_{s}^{n}\right)\right)$. Then, by (6.68), we obtain

$$
\begin{aligned}
T_{r}^{* *}\left(\varepsilon\left(\widehat{\mathbf{u}}_{1}\right)_{\mid \bar{\Omega}}\right) & =\operatorname{cl}_{\sigma\left(\mathbf{Y}^{1}(\bar{\Omega}), C_{\operatorname{div}}\left(\bar{\Omega}, \mathbf{E}_{s}^{n}\right)\right)} T_{r}\left(\varepsilon\left(\widehat{\mathbf{u}}_{1}\right)_{\mid \bar{\Omega}}\right) \\
& =\inf \left\{\operatorname{cl}_{\sigma\left(\widetilde{\mathbf{Y}}^{1}(\bar{\Omega}), C_{\operatorname{div}}\left(\bar{\Omega}, \mathbf{E}_{s}^{n}\right)\right)} \widetilde{T}_{r}(\mathbf{M}) \mid \mathbf{M} \in \widetilde{\mathbf{Y}}^{1}(\bar{\Omega})\right\} .
\end{aligned}
$$

For every $\mathbf{M} \in \mathbf{Y}^{1}(\bar{\Omega})$ we have $T_{r}(\mathbf{M})=\widetilde{T}_{r}(\mathbf{M})$. Hence for every $\mathbf{M} \in$ $\mathbf{Y}^{1}(\bar{\Omega})$ we get $\mathrm{cl}_{\sigma\left(\mathbf{Y}^{1}(\bar{\Omega}), C_{\mathrm{div}}\left(\bar{\Omega}, \mathbf{E}_{s}^{n}\right)\right)} T_{r}(\mathbf{M}) \geq \mathrm{cl}_{\sigma\left(\widetilde{\mathbf{Y}}^{1}(\bar{\Omega}), C_{\mathrm{div}}\left(\bar{\Omega}, \mathbf{E}_{s}^{n}\right)\right)} \widetilde{T}_{r}(\mathbf{M})$. Restriction of the measure $\varepsilon\left(\widehat{\mathbf{u}}_{1}\right)_{\mid \bar{\Omega}}$ to the open set $\Omega$ is denoted by $\varepsilon\left(\widehat{\mathbf{u}}_{1}\right)_{\mid \Omega}$. Because of (6.69) and (6.68), the point $\varepsilon\left(\widehat{\mathbf{u}}_{1}\right)_{\mid \bar{\Omega}}=\left(\varepsilon\left(\widehat{\mathbf{u}}_{1}\right)_{\mid \Omega},-\gamma_{B}^{I}\left(\widehat{\mathbf{u}}_{1}\right) \otimes_{s} \boldsymbol{\nu}\right) \in$ $\widetilde{\mathbf{Y}}^{1}(\bar{\Omega})$ is a minimum point of the function $\mathrm{cl}_{\sigma\left(\widetilde{\mathbf{Y}}^{1}(\bar{\Omega}), C_{\mathrm{div}}\left(\bar{\Omega}, \mathbf{E}_{s}^{n}\right)\right)} \widetilde{T}_{r}$ on the space $\widetilde{\mathbf{Y}}^{1}(\bar{\Omega})$. By $[12$, Chapter $1,(5.2)]$ we get $\mathbf{0} \in \partial\left(\operatorname{cl}_{\sigma\left(\widetilde{\mathbf{Y}}^{1}(\bar{\Omega}), C_{\mathrm{div}}\left(\bar{\Omega}, \mathbf{E}_{s}^{n}\right)\right)} \widetilde{T}_{r}\right)$ $\left(\varepsilon\left(\widetilde{\mathbf{u}}_{1}\right)_{\mid \bar{\Omega}}\right)$, where $\partial$ is a subgradient and $\mathbf{0} \in C_{\operatorname{div}}\left(\bar{\Omega}, \mathbf{E}_{s}^{n}\right)$. Then $\varepsilon\left(\widetilde{\mathbf{u}}_{1}\right)_{\mid \bar{\Omega}} \in$ $\partial\left(\operatorname{cl}_{\sigma\left(\widetilde{\mathbf{Y}}^{1}(\bar{\Omega}), C_{\mathrm{div}}\left(\bar{\Omega}, \mathbf{E}_{s}^{n}\right)\right)} \widetilde{T}_{r}\right)^{*}(\mathbf{0})$ (see [12, Chapter 1, Corollary 5.2]). By Corollary 4.1 of $\left[12\right.$, Chapter 1] we have $\varepsilon\left(\widehat{\mathbf{u}}_{1}\right)_{\mid \bar{\Omega}}=\left(\varepsilon\left(\widehat{\mathbf{u}}_{1}\right)_{\mid \Omega},-\gamma_{B}^{I}\left(\widehat{\mathbf{u}}_{1}\right) \otimes_{s} \boldsymbol{\nu}\right) \in$ $\partial\left(\widetilde{T}_{r}^{*}\right)(\mathbf{0})$. Then by $[12$, Chapter $1,(5.2)]$ we get

$$
\left\langle\left(\varepsilon\left(\widehat{\mathbf{u}}_{1}\right)_{\mid \Omega},-\gamma_{B}^{I}\left(\widehat{\mathbf{u}}_{1}\right) \otimes_{s} \boldsymbol{\nu}\right), \boldsymbol{\sigma}-\mathbf{0}\right\rangle_{1}+\widetilde{T}_{r}^{*}(\mathbf{0}) \leq \widetilde{T}_{r}^{*}(\boldsymbol{\sigma})
$$

for every $\boldsymbol{\sigma} \in C_{\operatorname{div}}\left(\bar{\Omega}, \mathbf{E}_{s}^{n}\right)$.

Step 2. Because of Assumption $4, \Gamma_{1}=\operatorname{Fr} \Omega \cap \mathcal{C}$, where $\mathcal{C}=\operatorname{clint} \mathcal{C} \subset \Omega_{1}$ is a closed Caccioppoli set and $d s(\operatorname{Fr} \Omega \cap \operatorname{Fr} \mathcal{C})=0$. Let $\mathcal{O}_{\Gamma_{0}}=\Omega_{1}-\mathcal{C}$. Then $d s\left(\Gamma_{0}-\left(\operatorname{Fr} \Omega \cap \mathcal{O}_{\Gamma_{0}}\right)\right)=0$ and $d s\left(\left(\operatorname{Fr} \Omega \cap \mathcal{O}_{\Gamma_{0}}\right)-\Gamma_{0}\right)=0$. We define $\Gamma_{0}^{\prime}=$ $\operatorname{Fr} \Omega \cap \mathcal{O}_{\Gamma_{0}}$. Then for every $k \in \mathbb{N}$ there exists an open set $\Omega_{k}^{\prime}$ such that $\Omega_{k}^{\prime} \subset$ $\mathcal{O}_{\Gamma_{0}}, \Omega_{k}^{\prime} \subset \subset \Omega_{1}, d x\left(\Omega_{k}^{\prime}\right)<1 /(2 k)$ and $\left\{x \in \Gamma_{0}^{\prime} \mid \boldsymbol{\gamma}_{B}^{I}\left(\widehat{\mathbf{u}}_{1}\right)(x) \neq \mathbf{0}\right\} \subset \Omega_{k}^{\prime}$.

Step 3. Suppose the singular part $\left(\varepsilon\left(\widehat{\mathbf{u}}_{1}\right)_{\mid \Omega}\right)_{s}$ is not 0 or $d s\left(\left\{x \in \Gamma_{0}^{\prime} \mid\right.\right.$ $\left.\left.\gamma_{B}^{I}\left(\widehat{\mathbf{u}}_{1}\right)(x) \neq \mathbf{0}\right\}\right)>0$. Then there exists $\zeta>0$ such that $\left\|\left(\varepsilon\left(\widehat{\mathbf{u}}_{1}\right)_{\mid \Omega}\right)_{s}\right\|_{\mathbb{M}_{b}}+$ $\int_{\Gamma_{0}^{\prime}}\left\|\left(\gamma_{B}^{I}\left(\widehat{\mathbf{u}}_{1}\right) \otimes_{s} \boldsymbol{\nu}\right)(x)\right\|_{\mathbf{E}_{s}^{n}} d s>\zeta$. Therefore, for every $k \in \mathbb{N}$, there exist open sets $\Omega_{k}^{\prime \prime} \subset \subset \Omega$ with $\Omega_{k}^{0} \equiv \Omega_{k}^{\prime \prime} \cup \Omega_{k}^{\prime} \subset \subset \Omega_{1}$ such that $d x\left(\Omega_{k}^{0}\right)<1 / k$ and $\left\|\left(\boldsymbol{\varepsilon}\left(\widehat{\mathbf{u}}_{1}\right)_{\mid \Omega_{k}^{\prime \prime}}\right)_{s}\right\|_{\mathbb{M}_{b}}+\int_{\Gamma_{0}^{\prime}}\left\|\left(\boldsymbol{\gamma}_{B}^{I}\left(\widehat{\mathbf{u}}_{1}\right) \otimes_{s} \boldsymbol{\nu}\right)(x)\right\|_{\mathbf{E}_{s}^{n}} d s>\frac{1}{2} \zeta$. The existence of the sequence $\left\{\Omega_{k}^{\prime \prime}\right\}_{k \in \mathbb{N}}$ satisfying the above conditions follows from the regularity of the measure $\boldsymbol{\varepsilon}\left(\widehat{\mathbf{u}}_{1}\right)_{\mid \Omega}$. By Assumption $5, B_{\mathbf{E}_{s}^{n}}\left(\boldsymbol{\sigma}_{L}(x), \delta_{0}\right) \subset \mathcal{K}(x)$ for every $x \in \Omega$. Then for every $k \in \mathbb{N}$ there exists $\varphi_{k} \in C_{0}^{1}\left(\Omega_{1}, \mathbf{E}_{s}^{n}\right)$ such that $\boldsymbol{\varphi}_{k \mid \Omega_{1}-\Omega_{k}^{0}}=0$,

$$
\left\|\boldsymbol{\varphi}_{k}(x)\right\|_{\mathbf{E}_{s}^{n}}<\frac{1}{2} \delta_{0}, \forall x \in \Omega_{k}^{0}
$$

and

$$
\left\langle\left(\varepsilon\left(\widehat{\mathbf{u}}_{1}\right)_{\mid \Omega},-\gamma_{B}^{I}\left(\widehat{\mathbf{u}}_{\mathbf{1}}\right) \otimes_{s} \boldsymbol{\nu}\right), \boldsymbol{\varphi}_{k \mid \bar{\Omega}}\right\rangle_{1}>\frac{1}{8} \zeta \delta_{0},
$$


since

$$
\left\|\left(\boldsymbol{\varepsilon}\left(\widehat{\mathbf{u}}_{1}\right)_{\mid \Omega_{k}^{\prime \prime}}\right)_{s}\right\|_{\mathbb{M}_{b}}+\int_{\Gamma_{0}^{\prime}}\left\|\left(\gamma_{B}^{I}\left(\widehat{\mathbf{u}}_{1}\right) \otimes_{s} \boldsymbol{\nu}\right)(x)\right\|_{\mathbf{E}_{s}^{n}} d s>\frac{1}{2} \zeta
$$

and

$$
\begin{aligned}
\left\|\varepsilon\left(\widehat{\mathbf{u}}_{1}\right)_{\mid \Omega_{k}^{0}}\right\|_{\mathbb{M}_{b}}=\sup \{ & \left\langle\varepsilon\left(\widehat{\mathbf{u}}_{1}\right)_{\mid \Omega_{k}^{0}}, \widetilde{\varphi}\right\rangle_{\mathbb{M}_{b} \times C\left(\Omega_{k}^{0}, \mathbf{E}_{s}^{n}\right)} \mid \\
\widetilde{\varphi} & \in C_{0}^{1}\left(\Omega_{k}^{0}, \mathbf{E}_{s}^{n}\right) \text { and } \forall x \in \Omega_{k}^{0},\|\widetilde{\varphi}(x)\|_{\left.\mathbf{E}_{s}^{n} \leq 1\right\} .}
\end{aligned}
$$

Step 4. By Assumption 3 for every $\widehat{r}>0$ there exists $\delta_{\widehat{r}}>0$ such that

$$
\left|j^{*}\left(x, \mathbf{w}_{1}^{*}\right)-j^{*}\left(x, \mathbf{w}_{2}^{*}\right)\right|<\delta_{\widehat{r}}\left\|\mathbf{w}_{1}^{*}-\mathbf{w}_{2}^{*}\right\|_{\mathbf{E}_{s}^{n}}
$$

for $d x$-a.e. $x \in \Omega$ and all $\mathbf{w}_{1}^{*}, \mathbf{w}_{2}^{*} \in \mathcal{K}(x)$ with $\left\|\mathbf{w}_{1}^{*}\right\|_{\mathbf{E}_{s}^{n}}<\widehat{r},\left\|\mathbf{w}_{2}^{*}\right\|_{\mathbf{E}_{s}^{n}}<\widehat{r}$. Then there exists $\delta>0$ such that

$$
\left|\widetilde{T}_{r}^{*}\left(\boldsymbol{\varphi}_{k \mid \Omega}\right)-\widetilde{T}_{r}^{*}(\mathbf{0})\right|<\frac{1}{2} \delta \delta_{0} \cdot d x\left(\Omega_{k}^{0} \cap \Omega\right)<\frac{1}{2} \delta \delta_{0} \frac{1}{k}
$$

for every $k \in \mathbb{N}$, since $\boldsymbol{\varphi}_{k}(x)+\boldsymbol{\sigma}_{L}(x) \in \mathcal{K}(x)$ for every $x \in \Omega$ and $\boldsymbol{\varphi}_{k \mid \Omega}+\boldsymbol{\sigma}_{L} \in$ $C\left(\bar{\Omega}, \mathbf{E}_{s}^{n}\right)(\mathrm{cf} .(6.73))$. By $(6.70)$ we get

$$
\left\langle\left(\varepsilon\left(\widehat{\mathbf{u}}_{1}\right)_{\mid \Omega},-\gamma_{B}^{I}\left(\widehat{\mathbf{u}}_{1}\right) \otimes_{s} \boldsymbol{\nu}\right), \boldsymbol{\varphi}_{k \mid \bar{\Omega}}\right\rangle_{1} \leq\left|\widetilde{T}_{r}^{*}\left(\boldsymbol{\varphi}_{k \mid \Omega}\right)-\widetilde{T}_{r}^{*}(\mathbf{0})\right|
$$

for every $k \in \mathbb{N}$. Then, due to (6.72) and (6.75), we have a contradiction, because $\frac{1}{2} \delta \delta_{0} \frac{1}{k} \rightarrow 0$ as $k \rightarrow \infty$.

\section{References}

[1] E. Acerbi, I. Fonseca and N. Fusco, Regularity results for equilibria in a variational model for fracture, Quaderni del Dipartimento di Matematica, Università degli Studi di Parma, March 1996, n. 126.

[2] G. Anzellotti, On the extremal stress and displacement in Hencky plasticity, Duke Math. J. 51 (1984), 133-147.

[3] G. Anzellotti and M. Giaquinta, Convex functionals and partial regularity, Arch. Rat. Mech. Anal. (3) 102 (1988), 243-272.

[4] A. C. Barroso, I. Fonseca and R. Toader, A relaxation theorem in the space of functions of bounded deformation, Ann. Scuola Norm. Sup. Pisa Cl. Sci. 29 (2000), 19-49.

[5] G. Bellettini, A. Coscia and G. Dal Maso, Compactness and lower semicontinuity properties in $S B D(\Omega)$, Math. Z. 228 (1998), 337-351.

[6] J. L. Bojarski, The relaxation of Signorini problems in Hencky plasticity, I : Threedimensional solid, Nonlinear Anal. 29 (1997), 1091-1116.

[7] G. Bouchitté, I. Fonseca and L. Mascarenhas, A global method for relaxation, Arch. Rat. Mech. Anal. 145 (1998), 51-98.

[8] G. Bouchitté and M. Valadier, Integral representation of convex functionals on a space of measures, J. Funct. Anal. 80 (1988), 398-420.

[9] A. Braides, A. Defranceschi and E. Vitali, A relaxation approach to Hencky's plasticity, Appl. Math. Optim. 35 (1997), 45-68. 
[10] M. Carriero, A. Leaci and F. Tomarelli, Free gradient discontinuities, in: Calculus of Variations, Homogenization and Continuum Mechanics, G. Bouchitté et al. (eds.), Ser. Adv. Math. Appl. Sci. 18, World Sci., Singapore, 1994.

[11] N. Dunford and J. T. Schwartz, Linear Operators, Part I, Interscience Publishers, New York, 1958.

[12] I. Ekeland and R. Temam, Convex Analysis and Variational Problems, NorthHolland, Amsterdam and New York, 1976.

[13] R. Engelking, General Topology, PWN-Polish Sci. Publ., Warszawa, 1977.

[14] A. Friedman and Y. Liu, Propagation of cracks in elastic media, Arch. Rat. Mech. Anal. 136 (1996), 235-290.

[15] M. Giaquinta, G. Modica and J. Souček, Cartesian Currents in the Calculus of Variations. Vol. II: Variational Integrals, Ergeb. Math. Grenzgeb. 38, Springer, Berlin, 1998.

[16] E. Giusti, Minimal Surfaces and Functions of Bounded Variation. lecture notes written by G. H. Williams, Dept. Math., Australian National Univ. 10, Canberra, 1977.

[17] R. Hardt and D. Kinderlehrer, Elastic plastic deformation, Appl. Math. Optim. 10 (1983), 203-246.

[18] R. Kohn and R. Temam, Dual spaces of stresses and strains with applications to Hencky plasticity, ibid. 10 (1983), 1-35.

[19] J. A. König, Shakedown of Elastic-Plastic Structures, PWN-Polish Sci. Publ., Warszawa, and Elsevier, Amsterdam, 1987.

[20] J. J. Moreau, Champs et distributions de tenseurs déformation sur un ouvert de connexité quelconque, Sém. d'Analyse Convexe, Univ. de Montpellier, 6 (1976).

[21] R. T. Rockafellar, Integral functionals, normal integrands and measurable selections, in: Nonlinear Operators and the Calculus of Variations, Lecture Notes in Math. 543, Springer, Berlin, 1975, 157-207.

[22] G. A. Seregin, Two dimensional variational problems in plasticity, Izv. Math. 60 (1996), 179-216.

[23] R. Temam, Mathematical Problems in Plasticity, Gauthier-Villars, Paris, 1985.

[24] R. Temam and G. Strang, Functions of bounded deformation, Arch. Rat. Mech. Anal. 75 (1980), 7-21.

[25] - - - Duality and relaxation in the variational problems of plasticity, J. Mécanique 19 (1980), 493-527.

[26] F. Tomarelli, Signorini problem in Hencky plasticity, Ann. Univ. Ferrara Sez. VII Sci. Mat. 36 (1990), 73-84.

Institute of Fundamental Technological Research

Polish Academy of Sciences

Świętokrzyska 21

00-049 Warszawa, Poland

E-mail: jbojar@ippt.gov.pl

Received on 19.8.2002;

revised version on 9.7.2003 